\title{
Pac1/LIS1 promotes an uninhibited conformation of dynein that coordinates its localization and activity
}

Matthew G. Marzo*, Jacqueline M. Griswold* and Steven M. Markus*ł

*Department of Biochemistry and Molecular Biology, Colorado State University, Fort Collins, Colorado, USA

${ }^{\ddagger}$ Corresponding author

Contact information for corresponding author:

Steven M. Markus

44 


\section{ABSTRACT}

46 Cytoplasmic dynein is a minus end-directed microtubule motor that transports myriad

47 cargos in various cell types and contexts. How dynein is regulated to perform all these

48 activities with a high degree of spatial and temporal precision is unclear. Recent studies

49 have revealed that human dynein-1 and dynein-2 can be regulated by a mechanism of

50 autoinhibition, whereby intermolecular contacts limit motor activity. Whether this

51 autoinhibitory mechanism is conserved throughout evolution, whether it can be affected

52 by extrinsic factors, and its precise role in regulating cellular dynein activity remain

53 unknown. Here, we use a combination of negative stain EM, single molecule motility

54 assays, genetic, and cell biological techniques to show that the autoinhibitory

55 conformation is conserved in budding yeast, and it plays an important role in

56 coordinating dynein localization and function in cells. Moreover, we find that the

57 Lissencephaly-related protein, LIS1 (Pac1 in yeast) plays an important role in regulating

58 this autoinhibitory conformation of dynein. Specifically, our studies demonstrate that

59 rather than inhibiting dynein motility, Pac1/LIS1 promotes dynein activity by stabilizing

60 the uninhibited conformation, which ensures appropriate localization and activity of

61 dynein in cells. 


\section{INTRODUCTION}

Cytoplasmic dynein is an enormous minus end-directed microtubule motor exceedingly complex in terms of its architecture, size, and reliance on accessories and regulators for proper activity. For instance, processive single molecule motility of human

67 dynein - itself comprised of 4 to 6 subunits - requires the 11 subunit dynactin complex in addition to an adaptor that links them together ${ }^{1,2}$. Although yeast dynein does not require dynactin for in vitro single molecule motility ${ }^{3}$, it does require this complex for in vivo activity ${ }^{4,5}$. Recent studies have yielded invaluable insight into the underlying reasons for the complexity of the dynein motor. For instance, the reliance on adaptors

72 (e.g., BicD2, Spindly, Hook3 ${ }^{1,2}$ ) to link dynein to dynactin ensures that cytoplasmic

73 dynein-1 - which effects motility of numerous and varied cargoes throughout the cell

74 cycle $^{6}$ - and dynactin are linked together at the right place (and presumably time) for

75 appropriate motility. Additionally, recent studies have revealed that dynactin helps to

76 orient the motor domains in a parallel manner that is conducive for motility ${ }^{7}$, thus

77 revealing the mechanistic basis for dynein's reliance on this large complex. Thus, the complexity of this molecular motor ensures that cargoes are transported to their target

79 destinations in accordance with the needs of the cell.

81 demonstrated that human dynein-1 and dynein-2 can also be auto-regulated by intra-

82 complex interactions. Specifically, intermolecular interactions between the motor

83 domains have been shown to stabilize an autoinhibited conformation of human dynein

84 called the phi particle (named for its resemblance to the Greek letter) ${ }^{7-10}$. In the case of 
85 dynein-2 (responsible for intraflagellar transport), the phi particle conformation - which

86 has been observed in its native context ${ }^{10}$ - reduces its velocity, ATPase activity and

87 microtubule landing rate ${ }^{9}$. Similarly, the autoinhibited dynein-1 conformation has been

88 shown to reduce its microtubule landing rate and motility properties ${ }^{7,11}$. Moreover, unlike

89 dynein-2 which is not regulated by dynactin ${ }^{12}$, uninhibited dynein-1 mutants interact

90 more readily with dynactin and the adaptor BicD2 ${ }^{7}$.

$91 \quad$ Although it is well established that human dynein adopts the autoinhibited phi

92 particle conformation (both dynein-1 and dynein-2), it is unclear if this conformational

93 state is evolutionarily conserved. Yeast dynein is of particular interest due to two

94 notable in vitro discrepancies with human dynein. In particular, unlike human dynein,

95 yeast dynein is processive in single molecule assays without the need for other factors,

96 such as dynactin ${ }^{3}$. The second notable feature that distinguishes yeast dynein is its

97 apparent ability to interact with dynactin in the absence of any additional factors (i.e.,

98 adaptors $)^{13}$. The reasons for these differences are unclear, but one possibility is that

99 yeast dynein does not adopt the autoinhibited phi particle conformation, which could

100 potentially account for its ability to walk in the absence of dynactin. This is supported by

101 studies showing that artificially separating the motor domains of human dynein-1 with a

102 rigid linker (thus preventing intermolecular contacts) is sufficient to convert it to a

103 processive motor ${ }^{11}$.

104 In addition to dynactin, the Lissencephaly protein LIS1 is another important

105 effector of dynein activity that is required for numerous dynein functions in cells ${ }^{14,15}$.

106 These include promoting dynein recruitment to various cellular sites ${ }^{16,17}$, and assisting in

107 dynein transport functions, including nuclear migration in neurons ${ }^{18,19}$, and high-load 
108 vesicular transport ${ }^{20-23}$. However, the mechanism by which LIS1 affects dynein, or dynein-dynactin activity remains controversial. For instance, in vitro studies have shown

110 that LIS1 can either reduce ${ }^{23-25}$ (for dynein alone) or increase dynein velocity (in the

111 context of intact dynein-dynactin-BicD2 complexes) ${ }^{25,26}$. In addition to promoting in vitro

112 force production by dynein ${ }^{23}$, studies have also shown that LIS1 can help in the initiation

113 of dynein-dynactin-BicD2 motility from the plus ends of dynamic microtubules ${ }^{25,27}$.

114 Studies with the budding yeast homolog of LIS1 - Pac1 - have shown that it reduces

115 the velocity of dynein motility ${ }^{28-31}$, presumably by uncoupling the ATPase cycle from the

116 conformational changes in the motor and microtubule-binding domains that elicit

117 microtubule release 29,30 . Thus, the precise role for Pac1/LIS1 in dynein function remains

118 confounded by these contrasting results. Although a role for Pac1/LIS1 in regulating the

119 autoinhibited conformation has not yet been reported, two studies found that LIS1 can

120 indeed promote dynein-dynactin interaction ${ }^{32,33}$, which is an expected consequence of

121 relieving dynein autoinhibition ${ }^{7}$.

122 Here, we set out to address the question of whether yeast dynein adopts an

123 autoinhibited phi particle conformation, and if so, what role it plays in regulating in vitro

124 and in vivo dynein activity. Our recent findings suggested the potential for yeast dynein

125 to adopt such a conformation ${ }^{34}$. Specifically, we found that engineering a neurological

126 disease-correlated mutation into yeast dynein leads to increased run lengths of single

127 molecules of dynein, and a localization pattern in cells that is suggestive of an

128 enhanced dynein-dynactin interaction. This mutation was within the linker domain - the

129 mechanical element responsible for the powerstroke ${ }^{35}$ - at a residue that was recently

130 shown to be important for maintenance of the phi particle conformation of human 
131 dynein ${ }^{7}$. Here, we use a combination of single particle analysis (by negative stain EM),

132 single molecule motility assays, genetic, and cell biological approaches to show that

133 yeast dynein indeed adopts a phi particle conformation that restricts its in vitro

134 processivity, and coordinates it localization and activity in cells. Moreover, we find that

135 Pac1 is an important regulator of the phi particle conformation. In particular, we found

136 that rather than inhibiting dynein motility, Pac1 promotes its activity by stabilizing the

137 'open', uninhibited conformational state. Our findings help explain recent observations

138 with LIS1 ${ }^{25,26}$, and support a model whereby Pac1/LIS1 is a key effector of dynein

139 autoinhibition.

141 RESULTS

142 Yeast dynein adopts an autoinhibited 'phi' particle conformation

143 We sought to determine whether yeast dynein adopts an autoinhibited

144 conformation (the 'phi' particle ${ }^{8}$ ). To this end, we developed a strategy to isolate

145 biochemical quantities of the intact yeast dynein complex that are of sufficient purity for

146 single particle analysis by negative stain electron microscopy. The yeast dynein

147 complex is comprised of light (Dyn2), light-intermediate (Dyn3), intermediate (Pac11),

148 and heavy chains (Dyn1) ${ }^{36}$. We generated a polycistronic plasmid containing all four

149 dynein complex subunits each flanked by a strong, galactose-inducible promoter

$150(G A L 1 p)$ on the $5^{\prime}$ end, and a synthetic transcriptional terminator $\left(\mathrm{T}_{\text {synth }}{ }^{37}\right)$ on the $3^{\prime}$

151 end. We included a tandem affinity tag (8His-ZZ, or "HZZ") followed by either a SNAP or

152 HALO tag on the N-terminus of Dyn1 for purification and fluorescent labeling of the

153 complex, respectively. In addition to these four gene cassettes, the plasmid also 

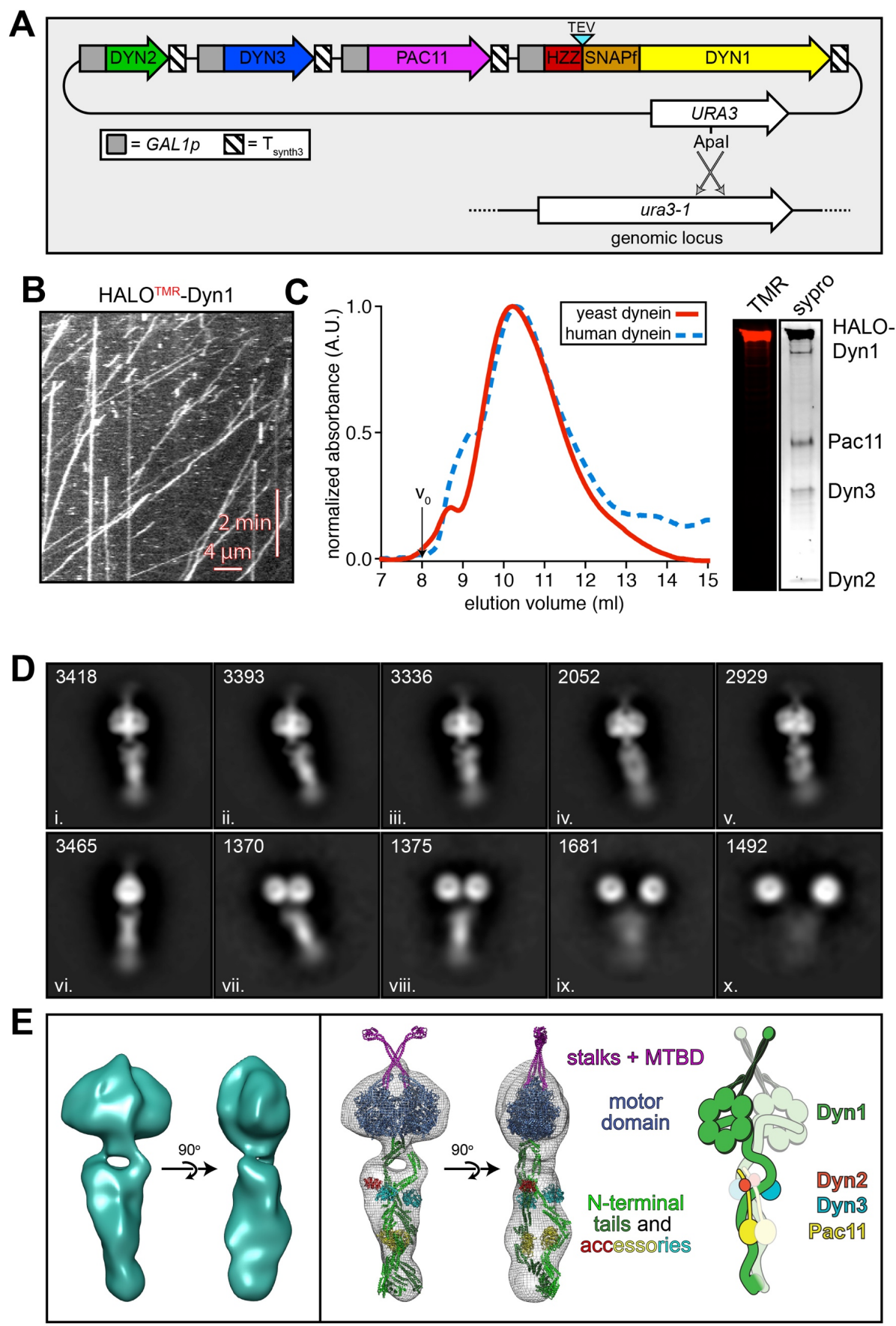

Figure 1. The yeast dynein complex adopts an autoinhibited phi particle conformation. (A) Schematic of the polycistronic plasmid used to produce the intact yeast dynein complex. Restriction digest with Apal (cuts within URA3 gene) targets the plasmid for homologous recombination into the ura3-1 locus as depicted. (B) Representative kymograph depicting single molecule motility of the purified overexpressed yeast dynein complex. (C) Elution profiles of yeast and human dynein complexes from Superose 6 resin (left), and scans of the same polyacrylamide gel depicting fluorescently labeled Dyn1 (via HaloTag-TMR) and the entire complex (via Sypro Ruby staining; right). (D) Representative negative stain EM class averages of the intact yeast dynein complex. Number of particles used to generate each class indicated in each panel. Classes $\mathrm{i}-\mathrm{vi}$ depict dynein in the autoinhibited, phi particle conformation, whereas vii $-x$ depict dynein in various open, uninhibited states. (E) 3D models of dynein in the autoinhibited state generated from 2D class averages with (right) and without (left) a high resolution 3D structure of human dynein-1 in the phi particle conformation (pdb 5NVU) manually docked into it. Note that the structures of the two tail domains have been slightly rotated with respect to the motor domains to better fit the 3D model, and that the structures of both TcTEX and Robl have been eliminated due to their absence from the yeast dynein complex. Also see Video S1. 
155 contains a URA3 cassette that provides a sequence for recombination-based genomic

156 integration (into the native ura3-1 allele) and a selectable marker (see Fig. 1A). Cells

157 with the plasmid integrated into their genome were grown in galactose-containing

158 media, and the dynein complex was subsequently isolated from cell lysates using either

159 tandem nickel/lgG affinity, or IgG affinity alone. We estimate the yield of the

160 overexpressed complex to be at least 50-fold greater than the non-overexpressed

161 complex. Single molecule motility assays confirmed the activity of the overexpressed

162 complex to be nearly identical to the non-overexpressed complex (Fig. 1B; also see Fig.

163 4B and 5). Importantly, the increased yield permitted us to isolate the complex to a high

164 degree of purity using size exclusion chromatography, which revealed an elution profile

165 nearly identical to the human dynein complex isolated from insect cells (Fig. 1C).

166 With the high purity dynein complex in hand, we used negative stain electron

167 microscopy to obtain the first ever high magnification view of the intact yeast dynein

168 complex. This revealed the presence of several conformational states, including those

169 in an open, uninhibited state (Fig. S1A, green arrow), and those in an apparent phi

170 particle conformation (Fig. S1A, red arrows), with the large majority being in the latter

171 state (Fig. S1B). Reference-free 2D class averages provided images that appear

172 strikingly similar to the intact human dynein-1 complex ${ }^{1,7}$, and to an artificially dimerized

173 motor domain truncation of dynein-2 in a phi particle conformation ${ }^{9}$ (Fig. 1D).

174 Specifically, the N-terminal tail domains - which exhibit flexibility with respect to the

175 motor domains - appear to be twisted around one another, which we confirmed by

176 generating three-dimensional reconstructions (Fig. 1E, left, and Video S1).

177 Intermolecular contacts appear to extend to the motor domains and the coiled-coil 
178 stalks, which connect the AAA ring to the microtubule-binding domains (MTBDs). Much

179 like the human dynein-1 and dynein-2 structures, the stalks cross each other in an " $X$ "-

180 like configuration in a manner that seems contrary to motility. We confirmed the high

181 degree of similarity between the human and yeast dynein-1 phi particle conformations

182 by manually docking a high resolution cryo-EM structure of human dynein (pdb 5NVU ${ }^{7}$ )

183 into our 3D model (Fig. 1E, right, and Video S1).

184 Of note, previous observations of an artificially-dimerized (via glutathione S-

185 transferase, GST), truncated yeast dynein motor domain fragment (lacking the $\mathrm{N}$ -

186 terminal tail domain) revealed a lack of phi particle conformations. This includes

187 observations by negative stain $\mathrm{EM}^{28}$, and within the crystal lattice of the crystalized

188 motor domain fragment ${ }^{38,39}$. Thus, in contrast to the dynein-2 isoform of dynein, for

189 which the motor domain is sufficient to adopt the phi particle conformation (as apparent

190 in the crystal lattice and by negative stain $E \mathrm{M}^{9,40}$ ), yeast dynein requires the tail domain

191 for assembly of this autoinhibited conformation. It is interesting to note that several of

192 our class averages appear to depict a conformation in which the motor domains are

193 closely apposed, but unbound, and the tails appear to be wrapped around one another

194 (Fig. 1D; classes vii and viii). Taken together, this suggests that contact points within the

195 tail domain provide important contacts that likely stabilize the autoinhibited

196 conformation.

198 Disruption of the autoinhibited conformation leads to increased single molecule

199 processivity

We previously found that a disease-correlated amino acid substitution within the

201 linker domain - the mechanical element responsible for the powerstroke - leads to an 
202 increase in single molecule run length ${ }^{34}$. This residue (K1475) is equivalent to one

203 known to stabilize the autoinhibited conformation of human dynein $(\mathrm{R} 1567)^{7}$. If the

204 increased processivity is a consequence of disrupted phi particle formation, then we

205 reasoned that mutations at other potential phi particle interfaces would also lead to

206 increased run lengths. A high resolution cryo-EM structure of the human dynein phi

207 particle identified four distinct surfaces that comprise the inter-molecular interface

208 (linker-linker, linker-AAA4, AAA5-AAA5, and stalk-stalk; Fig. 2A) ${ }^{7}$. Given the apparent

209 similarities between the yeast and human dynein phi particles (see Fig. 1E and Video

210 S1), we wondered whether the intermolecular contact points were conserved, and if so,

211 what effect disrupting the phi particle has on dynein motility.

212 Sequence alignment and homology modeling of pertinent regions of Dyn1 (those

213 at the intermolecular interface) into the high resolution cryo-EM structure of the human

214 dynein phi particle $\left(\mathrm{pdb}_{5} \mathrm{NVU}^{7}\right)$ revealed a high degree of conservation at the four

215 intermolecular surfaces (Fig. 2A). We focused initially on the linker-AAA4 interface (Fig.

$2162 \mathrm{~A}$, surface 2), which is presumably stabilized in part by electrostatic interactions

217 between negatively charged D2868, and positively charged K1475 and K1517. We

218 found that substituting either positively charged residue (K1475 or K1517) with a

219 negatively charged residue (glutamate) resulted in similar increases in single molecule

220 processivity (from $2.0 \mu \mathrm{m}$ to $3.4 \mu \mathrm{m}$ and $3.3 \mu \mathrm{m}$, respectively; $\mathrm{p}<0.0001$ ), while

221 eliminating both (K1475E K1517E) led to an even greater increase in run length (to 5.8

$222 \mu \mathrm{m}$; Fig. 2B). Consistent with these residues' role in stabilizing the linker-AAA4

223 interface, substituting the negatively charged D2868 for a positively charged one

224 (D2868K) led to an increase in run length that was statistically indistinguishable from the 

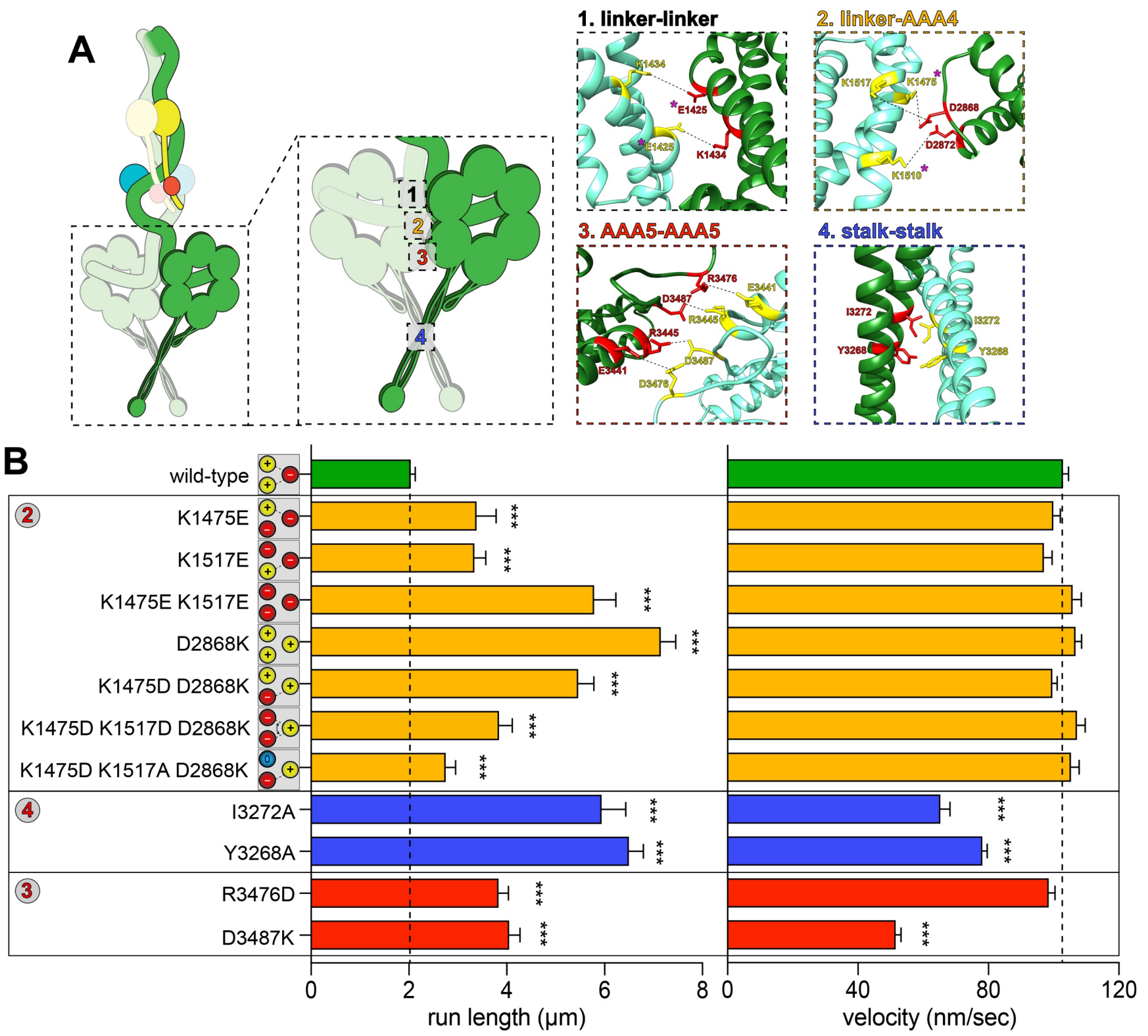

Figure 2. Disrupting phi particle contact points extends single molecule run lengths. (A) Cartoon depicting four predicted intermolecular contact surfaces within the motor domains that stabilize the phi particle conformation. Four insets show respective regions of yeast dynein modeled into human dynein phi particle structure. Structural models were generated using one-to-one threading of the yeast $D Y N 1$ sequence into $5 \mathrm{NVU}^{7}$ on the Phyre2 server ${ }^{72}$. Residues with magenta asterisks are mutated in patients suffering from neurological disease $^{62-64}$ (see Discussion). (B) Single molecule run length (from fitting of raw data to one-phase decay) and velocity values for wild-type and mutant dyneins with phi particle disrupting mutations (at surfaces 2, 3 and 4 , as indicated). Cartoons along vertical axis depict electrostatic interactions (or lack thereof) among residues 1517, 1475 (left circles) and 2868 (right circle) at linker-AAA4 surface. Note that the degree of processivity enhancement is inversely proportional to the number of charge interactions at this surface. Error bars indicate standard error (between 150 - 528 motors from at least two independent experiments were analyzed for each). Statistical significance was determined using a Mann-Whitney test (for run length) or with an unpaired Welch's $t$ test (for velocity; ${ }^{* * *}, p \leq 0.0001$ ). Also note that we generated and tested the motility of two other point mutants at interface 3, E3441K and R3445D, both of which were inactive in single molecule assays (not shown). 
226 K1475E K1517E double mutant $(7.2 \mu \mathrm{m} ; \mathrm{p}=0.246)$. Interestingly, we were able to

227 reduce these run length values by substituting back residues that would be predicted to 228 replace the broken electrostatic pairing (K1475D D2868K; K1475D K1517A D2868K; or, 229 K1475D K1517D D2868K). These results indicate that the linker-AAA4 interface is 230 indeed important for assembly of the autoinhibited conformation of yeast dynein, which 231 attenuates single molecule processivity of the intact complex. We next wondered if other predicted interfaces affect formation of the 233 autoinhibited conformation, and what role they play in affecting dynein motility.

234 Consistent with the apparent interaction surface observed in our 2D averages within the 235 stalk (Fig. 2A, surface 4; see Fig. 1D, classes i - v), substitution of either Y3268 or I3272 236 with an alanine led to an increase in run length comparable to those mutants lacking all 237 electrostatic contacts at the linker-AAA4 interface (Fig. 2B). Unlike the linker-AAA4 238 interface mutants, both Y3268A and I3272A exhibited reductions in velocity values 239 (from $102.8 \mathrm{~nm} / \mathrm{sec}$ to $73.2 \mathrm{~nm} / \mathrm{sec}$ for $Y 3268 \mathrm{~A}$, or $78.4 \mathrm{~nm} / \mathrm{sec}$ for I3272A; $\mathrm{p}<0.0001$ ).

240 This could be due to disrupted kinetics of helix sliding in the coiled coil stalk (i.e.,

241 changes in the heptad registry), which is responsible for communicating nucleotide-

242 dependent conformational changes within the motor domain to the microtubule-binding 243 domain ${ }^{40-42}$. Finally, charge reversal substitutions at either R3476 (to an aspartate) or 244 D3487 (to a lysine) at the AAA5-AAA5 interface (Fig. 2A, surface 3) also led to 245 increases in run length (from $2.0 \mu \mathrm{m}$ to 3.8 and $4.0 \mu \mathrm{m}$, respectively; $\mathrm{p}<0.0001$ ) 246 comparable to the single charge substitution mutants at the linker-AAA4 interface.

247 Although the R3476D mutant exhibited normal velocity, the D3487K substitution 248 reduced dynein velocity to approximately half that of wild-type. Taken together, these 
249 findings confirm the conserved nature of the intermolecular contact points that stabilize

250 the autoinhibited state of yeast dynein. They also indicate that the ability to adopt the

251 phi particle conformation is sufficient to severely limit the processivity of the yeast

252 dynein complex, which, in the absence of phi particle formation, can achieve run lengths

253 that match that of the human dynein-dynactin-BicD2 complex $\left(7.2 \mu \mathrm{m}\right.$ for dynein ${ }^{\mathrm{D} 2868 \mathrm{~K}}$

254 versus 5 - $9 \mu \mathrm{m}$ for human dynein-dynactin-BicD2) ${ }^{1,2,43}$. It is interesting to note that the

255 minimal, GST-dimerized dynein fragment exhibits run lengths (1.6 $\mu \mathrm{m}$; see Fig S5C)

256 much lower than that achievable by the phi particle disrupting mutants (as high as 7.2

$257 \mu \mathrm{m}$, or 4.5-fold higher), in spite of this fragment not adopting the phi particle

258 conformation. This indicates that the native tail domain permits an arrangement of the

259 motor domains that is much more conducive to processive motility than the GST moiety.

261 Dynein autoinhibition restricts cortical localization

Although preventing human dynein from adopting the autoinhibited conformation

263 by mutagenesis had no apparent effect on processivity, it did in fact lead to a significant

264 enhancement in the ability of dynein to interact with dynactin and the adaptor BicD2 ${ }^{7}$.

265 Previous studies have shown that dynactin is required for localization of dynein to

266 cortical Num1 receptor sites, but not to microtubule plus ends ${ }^{4,31,44}$ (Fig. 3A). Moreover,

267 in instances when dynactin interaction with dynein is enhanced ${ }^{45,46}$, the number of

268 dynein molecules found at cortical sites increases ${ }^{46}$, and dynein offloading to the cell

269 cortex becomes apparent from live cell imaging ${ }^{31}$. In light of the limiting nature of

270 dynactin at microtubule plus ends (1 dynactin complex for every 3 dynein complexes at

271 a plus end $)^{46}$, these observations suggest that interaction with dynactin is a limiting step 
A

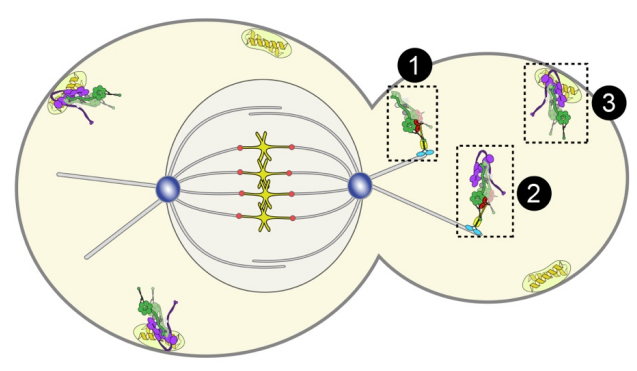

plus end dynein:

- dynactin-independent - Pac1-dependent

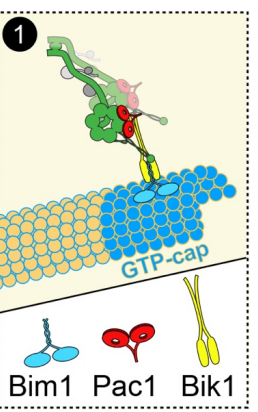

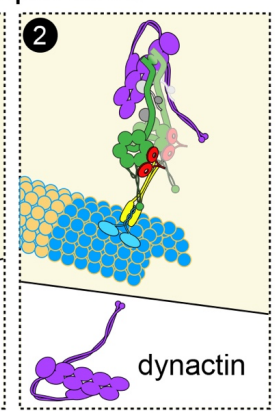

cortical dynein: - dynactin-dependent -Pac1-dependent

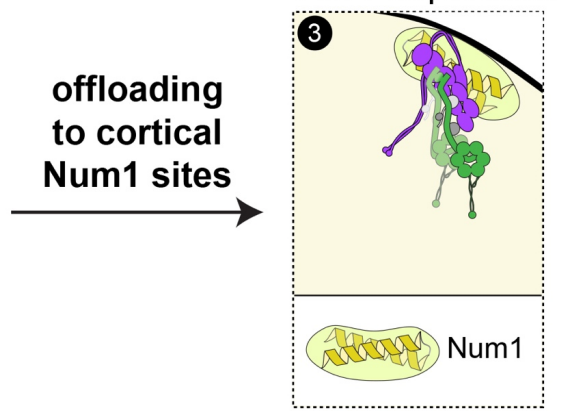

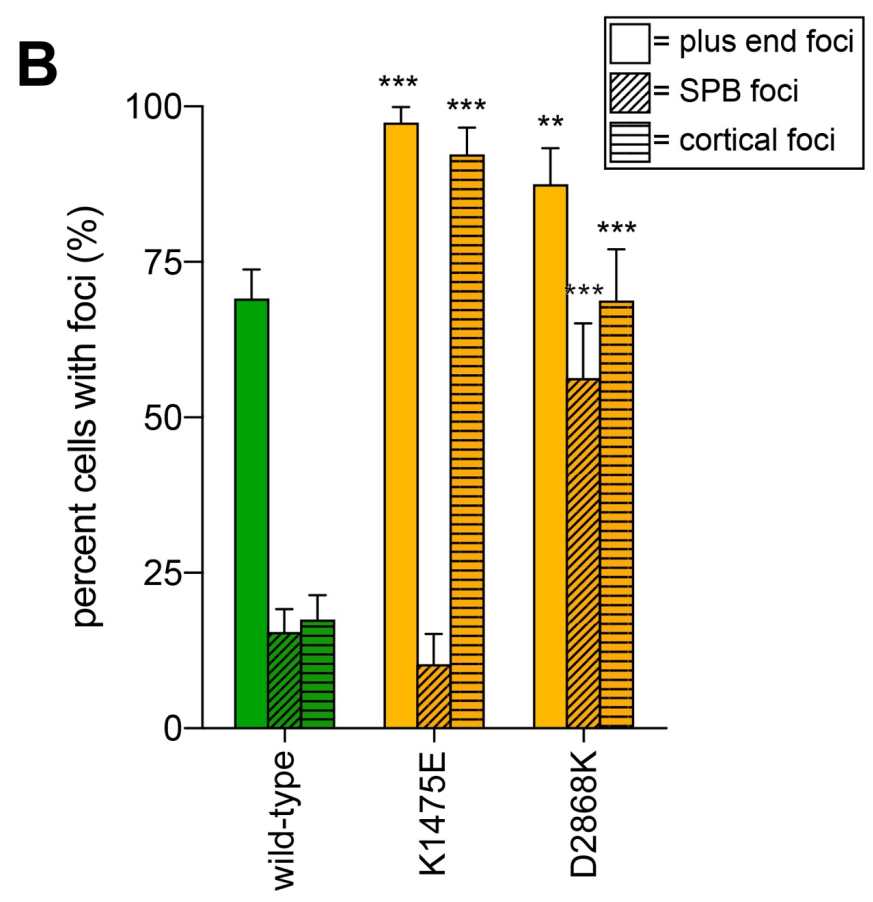
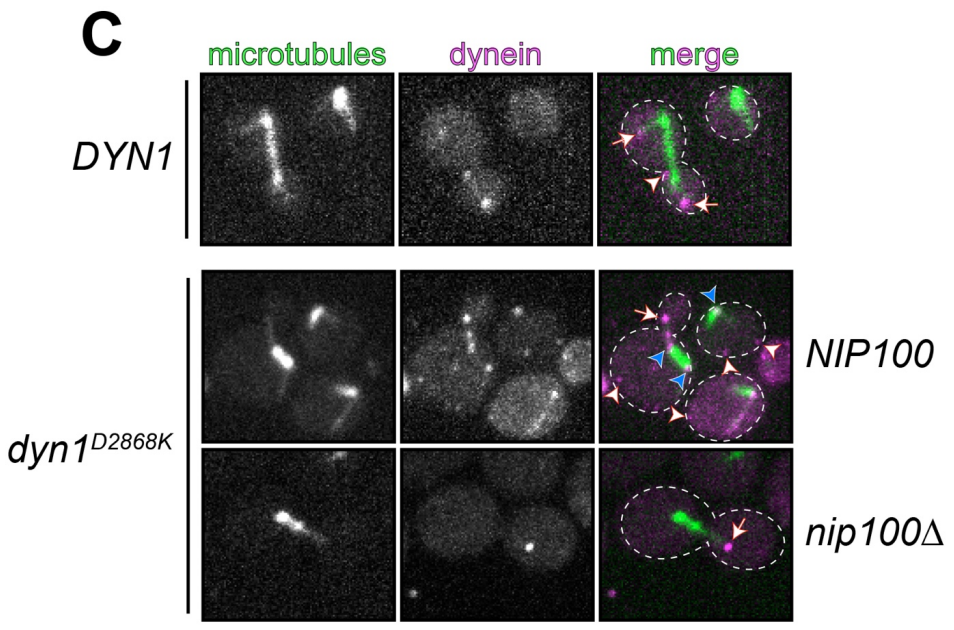

Figure 3. The autoinhibited conformation restricts plus end and cortical localization of dynein. (A) Cartoon depicting the two main sites of dynein localization (microtubule plus ends, and cell cortex), and the molecular requirements for each. Dynein plus end localization (1) requires Bik $1^{57}$ and Pac $1^{44}$, with Bim1 potentially playing some role in this process, but does not require dynactin ${ }^{4}$. Rather, dynactin plus end localization (2) relies on dynein ${ }^{4}$. Subsequent to plus end targeting, dynein-dynactin complexes are offloaded to cortical Num1 sites ${ }^{31,73}$ (3). (B) Plot depicting the frequency of plus end, SPB (spindle pole body) and cortical targeting for wild-type and mutant Dyn1 ( $n \geq 32$ mitotic cells for each). Error bars indicate standard error of proportion. Statistical significance was determined by calculating Z scores (see Methods). (C) Representative images of wild-type or mutant dynein (D2868K) localizing in otherwise wild-type or nip100 $\Delta$ (dynactin component) cells. Note the lack of cortical localization of Dyn1 ${ }^{\mathrm{D286K}}$ in nip $100 \Delta$ cells (white arrowheads, cortical foci; white arrows, plus end foci; blue arrowheads, SPB foci). 
273 in the delivery of dynein to cortical Num1 sites. Thus, if disruption of the autoinhibited

274 conformation of yeast dynein leads to enhanced dynactin interaction, then we expect to

275 see an increased frequency of dynein cortical localization.

276 To determine whether this was the case, we compared the localization pattern of

277 wild-type Dyn1-3GFP to that of the K1475E and D2868K mutants, which exhibited

278 modest and strong in vitro processivity phenotypes, respectively (see Fig. 2B).

279 Consistent with the notion that disrupting the phi particle promotes interaction with

280 dynactin, we observed a large increase in the number of cells exhibiting cortical dynein

281 foci (Fig. 3B and C). Although D2868K had a stronger processivity phenotype in the

282 single molecule assay, it did not exhibit a stronger cortical localization phenotype than

283 the K1475E mutant. However, we did note that the D2868K cells possessed a higher

284 frequency of dynein foci associated with the spindle pole bodies (SPBs), where the

285 minus ends of microtubules are anchored. Although the relevance of SPB foci is not

286 entirely clear, we previously noted that dynein complexes accumulated at this site when

287 they were activated by overexpression of a Num1 coiled-coil-containing fragment ${ }^{47}$.

288 Thus, the SPB pool of dynein molecules might represent "activated" dynein motors. As

289 the D2868K mutant exhibited a more robust in vitro processivity phenotype than

$290 \mathrm{~K} 1475 \mathrm{E}$, the former mutation likely results in fewer inhibited dynein molecules in cells.

291 Thus, the increased SPB localization of D2868K is likely a consequence of its increased

292 activity. Finally, we confirmed that dynactin was indeed required for the cortical

293 localization of Dyn1 $1^{\mathrm{D} 2868 \mathrm{~K}}$ by imaging its localization in cells lacking the dynactin

294 component, Nip100 (Fig. 3C, nip1004). These data are consistent with the notion that 
295 the phi particle restricts dynein-dynactin interaction, which limits association with cortical

296 Num1 receptor sites.

298 Peptide insertion between motor and tail domains ablates phi particle conformation

In addition to an increase in cortical localization, we also noted that the K1475E and D2868K mutants localized to microtubule plus ends to a greater extent than wild-

302 type dynein (see Fig. 3B; $p \leq 0.040$ ). We previously noted that the frequency of plus end 303 localization - which is Pac1-dependent ${ }^{44}$ (see Fig. 3A) - is increased for a truncated 304 dynein motor domain fragment (Dyn1 $\left.1_{\text {MOTOR }}\right)^{48}$, and also for a dynein mutant in which a

305 helical linker peptide (helical linker 3, HL3) was inserted between the tail and motor 306 domains (Dyn1 HL3; $_{3}$ see Fig. 4A) ${ }^{31}$. Of note, Dyn1 ${ }_{\mathrm{HL} 3}$ also localizes to the cell cortex to a 307 greater extent than wild-type Dyn $1^{31}$, much like an isolated tail domain fragment 308 (Dyn1 TAIL $^{48}$. We originally generated the Dyn1 ${ }_{\mathrm{HL} 3}$ mutant to test the hypothesis that the 309 motor domain plays a direct role in restricting the

310 cortical localization capacity of Dyn1 TAIL ${ }^{31}$. We predicted that insertion of the HL3

311 peptide would sufficiently separate the tail and motor domains such that the motor

312 domain would no longer be able to block the tail domain's interaction with cortical Num1

313 receptors (i.e., by "unmasking" the tail domain; see Fig. 4A, "original model”). Although

314 our hypothesis was indeed supported by localization data ${ }^{31}$, there has been no

315 structural or biochemical evidence to support our proposed mechanism of motor-based

316 inhibition of the tail domain. Thus, we wondered whether the HL3 insertion simply 
bioRxiv preprint doi: https://doi.org/10.1101/684290; this version posted June 27, 2019. The copyright holder for this preprint (which was not certified by peer review) is the author/funder. All rights reserved. No reuse allowed without permission.

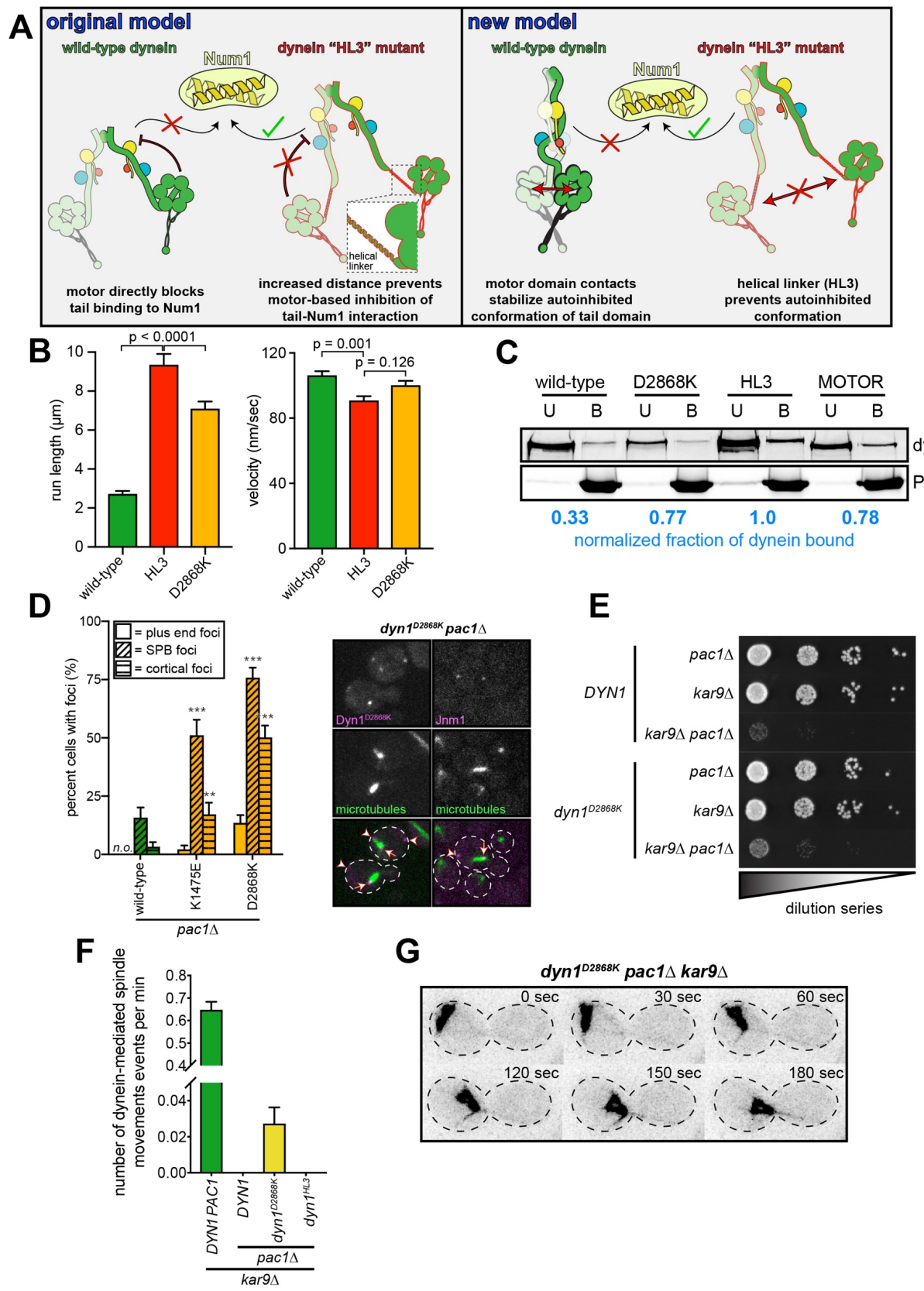

Figure 4. Release of dynein autoinhibition permits Pac1/LIS1-independent localization and function. (A) Cartoons depicting original, and new models accounting for "unmasking" phenotype observed with Dyn $1^{\mathrm{HL} 3}$ mutant ${ }^{31}$. Wild-type dynein tail domain is unable to associate with Num1 in the absence of plus end-targeting; however, addition of helical linker 3 (HL3) between tail and motor domains permits dynein to associate with Num1 independent of plus end-targeting. Our original model posited that this was a consequence of the motor domain directly precluding the tail domain from contact Num1; however, our new model is that contacts within the motor domain stabilize the phi particle conformation, in which the tail domains are in a twisted state that is unable to interact with Num1. In this latter model, insertion of HL3 prevents the adoption of the phi particle conformation. (B) Single molecule run length (from fitting of raw data to one-phase decay) and velocity values for wild-type and indicated mutant dyneins, as indicated, purified using plasmid-integration strategy described in Figure $1 \mathrm{~A}$ ( $n \geq 224$ motors for each, from at least two independent experiments; error bars indicate standard error). Statistical significance was determined using a Mann-Whitney test (for run length) or with an unpaired Welch's t test (for velocity). (C) Bead binding assay illustrating increased affinity of Pac1 for "open" dyneins (dynein ${ }^{\mathrm{D} 286 \mathrm{~K}}$ and GST-dynein ${ }_{\text {MотоR }}$ ). Purified dyneins were incubated with Pac1-FLAG-SNAP-decorated beads, and the bound ("B") and unbound ("U") fractions were resolved by SDS-PAGE. The normalized, relative bound and unbound fractions were determined by measuring band intensities. (D) Plot depicting the fraction of cells with indicated mutant or wild-type Dyn1 foci in pac1 $\Delta$ cells (n $\geq 34$ mitotic cells for each; "n.o.", none observed; error bars indicate standard error of proportion). Representative fluorescence images depicting the presence of cortical dynein (Dyn1) and dynactin (Jnm1) in dyn1 ${ }^{\text {D2868K }}$ cells (arrowheads, cortical foci; arrows, SPB foci). Statistical significance was determined by calculating $Z$ scores $\left({ }^{* *}, p=0.011 ;{ }^{* * *}, p \leq 0.0001\right)$. (E) Serial dilutions of cells with indicated genotype were plated on rich media (YPA supplemented with $2 \%$ glucose) and grown at $30^{\circ} \mathrm{C}$ for 2 days (as shown) or 4 days (see Fig. S3A). Note the partial rescue of cell viability in kar9s pac1 $\Delta$ dyn $1^{\text {D2868K }}$ cells as compared to $D Y N 1$ kar9 $\Delta$ pac1 $1 \Delta$ cells. $(F)$ Plot depicting number of dynein-mediated spindle movements per cell per minute in hydroxyurea (HU)-arrested cells (all of which are kar9s; see Methods; $\mathrm{n} \geq 32 \mathrm{HU}$ arrested cells for each). (G) Representative time-lapse fluorescence images of a hydroxyurea (HU)-arrested dyn1 ${ }^{\text {22868K }}$ pac1 $1 \Delta$ kar9 $\Delta$ cell exhibiting a dynein-mediated spindle movement. 
319 disrupts the phi particle conformation (Fig. 4A, "new model"), which would result in an

320 enhanced interaction between dynein, dynactin and Num1.

321 Single molecule analysis of Dyn1 $1_{\mathrm{HL} 3}$ revealed that this mutant exhibits run

322 lengths that exceed all other phi particle disrupting mutants $(9.3 \mu \mathrm{m} ; \mathrm{p} \leq 0.0001)$,

323 suggesting that HL3 peptide insertion indeed disrupts phi particle formation more than

324 any of the single point mutants (Fig. 4B). Since we expressed the HL3 mutant complex

325 using the strategy described in Figure 1, we compared its motility to similarly isolated

326 wild-type and D2868K complexes. We noted that although dynein ${ }^{\mathrm{HL} 3}$ and dynein ${ }^{\mathrm{D} 2868 \mathrm{~K}}$

327 exhibited significantly longer run lengths than wild-type dynein $(p<0.0001)$, the

328 overexpressed wild-type dynein complex exhibited slightly longer runs than the non-

329 overexpressed complex $(2.0 \mu \mathrm{m}$ versus $2.7 \mu \mathrm{m}, \mathrm{p}=0.016$; see Fig. S2A). The same

330 was not true for dynein ${ }^{\mathrm{D} 2868 \mathrm{~K}}(7.2 \mu \mathrm{m}$ for native, versus $7.3 \mu \mathrm{m}$ for overexpressed; $\mathrm{p}=$

331 0.9488). This suggests that the phi particle conformation of the overexpressed dynein

332 complex is more labile than the non-overexpressed complex. Consistent with this

333 notion, the run length of the overexpressed wild-type complex - but not the one

334 expressed from native promoters - increased over time to similar levels as the D2868K

335 mutant, even when stored at $-80^{\circ} \mathrm{C}$ (Fig. S2; also see below). By comparison, we only

336 observed a minor increase over time for the non-overexpressed wild-type complex, and

337 for the overexpressed dynein ${ }^{\mathrm{D} 2868 \mathrm{~K}}$. This is similar to observations with human dynein,

338 which needs to be prepared fresh in order to obtain a sufficient proportion of phi

339 particles ${ }^{7}$.

340 We previously noted that, like the truncated motor domain fragment ${ }^{48}$ (both the 341 monomeric $^{48}$ and artificially dimerized ${ }^{3}$ variants) - which does not adopt the phi particle 
342 conformation (see above) - the Dyn1 $1_{\mathrm{HL} 3}$ mutant exhibits higher affinity for Pac1 than

343 wild-type dynein ${ }^{31}$. If this increased affinity is a consequence of Pac1 preferentially

344 binding to an 'open', uninhibited dynein conformation, then we reasoned that a

345 disrupted phi particle mutant would also exhibit higher affinity for Pac1 than wild-type

346 dynein. To test this, we assessed the degree of dynein-Pac1 binding using a co-

347 pelleting assay. Either wild-type or dynein ${ }^{\mathrm{D} 2868 \mathrm{~K}}$ was incubated with Pac1-FLAG-SNAP-

348 decorated beads, and the bound and unbound fractions were quantitatively compared.

349 As a control, we also included GST-dyneinмотоR, which we expected to exhibit high

350 Pac1 affinity ${ }^{3,48}$. Consistent with the notion that Pac1 preferentially binds to dynein in its

351 'open', uninhibited conformation, we found that dynein ${ }^{\mathrm{D} 2868 \mathrm{~K}}$ and GST-dynein ${ }_{\text {MOTOR both }}$

352 exhibited higher affinity for Pac1 than wild-type dynein (Fig. 4C). This also indicates that

353 the likely reason for the altered localization ${ }^{31}$ and single molecule behavior of dynein ${ }^{\mathrm{HL} 3}$

354 is that it is in a constitutively uninhibited state.

Disruption of the phi particle leads to Pac1-independent cortical dynein activity

Given the phenotypic similarities between the phi particle disrupting mutants and property of the phi-disrupting mutants. Specifically, we previously found that the HL3 peptide insertion permits the dynein complex to bypass the need for Pac1 for delivery to

361 cortical Num1 receptor sites ${ }^{31}$. To determine the role of Pac1 in localizing phi particle

362 disrupting mutants to various sites in cells, we imaged Dyn1-3GFP (wild-type or mutant)

363 in cells lacking Pac1 (pac1 1 ). Consistent with prior observations ${ }^{44,48}$, Pac1 was required

364 for normal plus end and cortical localization of wild-type dynein (Fig. 4D). This is 
365 consistent with the offloading model for dynein function, in which dynein first associates

366 with microtubule plus ends, from where it is delivered - or offloaded - to cortical Num1

367 sites $^{31,49}$. Surprisingly, both Dyn1 $1^{\mathrm{K} 1475 \mathrm{E}}$ and Dyn1 ${ }^{\mathrm{D} 2868 \mathrm{~K}}$ were capable of localizing to the

368 cell cortex in the absence of Pac1 (Fig. 4D). The frequency of Dyn1 ${ }^{\mathrm{D} 2868 \mathrm{~K}}$ cortical

369 localization in pac1 $(50 \%)$ cells was similar to that of Dyn1 $1_{\mathrm{HL} 3}$ (as noted previously ${ }^{31}$,

$370 \sim 45 \%$ of cells exhibit cortical Dyn $1_{\mathrm{HL} 3}$ foci in pac1 $1 \Delta$ cells).

371 We wondered if these Pac1-independent cortical pools of dynein were functional.

372 To assess this, we performed a highly sensitive in vivo activity assessment, in which

373 dynein-mediated spindle movements are visualized and quantitated ${ }^{5}$. To eliminate any

374 dynein-independent contributions to spindle movements, these assays were performed

375 in cells lacking Kar9, a protein that is required for an actin/myosin-mediated spindle

376 orientation pathway ${ }^{50-52}$. Generation of yeast strains lacking Kar9 and dynein pathway

377 components also permits a genetic assessment of dynein functionality. In particular,

378 whereas cells deleted for either dynein or Kar9 pathway components exhibit no

379 apparent growth phenotypes, combined deletion of any of the genes involved in these

380 two pathways results in significant synthetic growth defects ${ }^{53}$. As shown in Figure 4E,

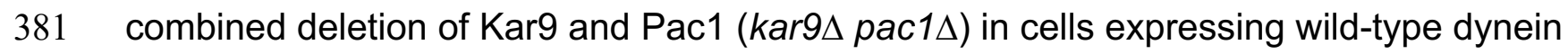

382 leads to severe growth defects. Interestingly, pac1 $1 \Delta$ kar9 $\Delta$ cells expressing Dyn $1^{\mathrm{D} 2868 \mathrm{~K}}$

383 exhibited growth defects less severe than those expressing wild-type dynein,

384 suggesting that the D2868K mutation partly rescues the loss of Pac1 (Fig. 4E and Fig.

385 S3A). Interestingly, we did not note a similar rescue for cells expressing Dyn1 ${ }^{\mathrm{HL} 3}$ (Fig.

386 S3B), suggesting that, although this mutant can bypass Pac1 for cortical localization ${ }^{31}$, 
387 and it is a highly processive motor in vitro, it is unable to move the mitotic spindle in 388 cells.

Consistent with the need for Pac1 for cortical localization of wild-type dynein, we

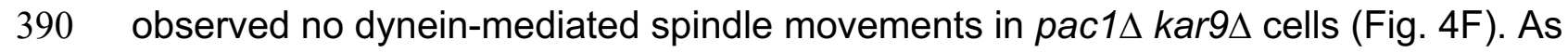

391 expected from the synthetic growth defects in dyn $1^{H L 3}$ kar9 $\Delta$ cells, we also observed no

392 dynein-mediated spindle movements in these cells. Surprisingly, we did in fact observe

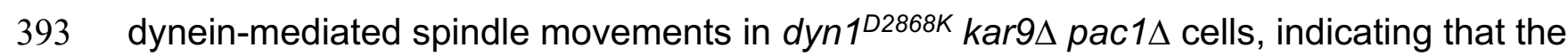

394 Pac1-independent cortical populations of the uninhibited dynein mutant are indeed

395 active (Fig. 4F and G). Given the ability of the uninhibited dynein mutant to rescue the

396 loss of Pac1, this suggests that at least one function of Pac1 is to release dynein from

397 its autoinhibited phi particle conformation.

\section{Pac1 stabilizes the uninhibited conformation of motile dynein complexes}

To gain additional insight into the potential mechanism by which Pac1 may be

401 affecting dynein autoinhibition we studied available structural information. Specifically,

402 we focused on two structures: a monomeric yeast dynein motor domain bound to

$403 \operatorname{Pac}^{30}$ (with Pac1 bound to a conserved site on the dynein motor domain ${ }^{26}$ ), and the

404 human dynein complex in the autoinhibited state ${ }^{7}$. Docking of the Pac1-bound motor

405 domain into one of the two motors in the autoinhibited state revealed an apparent steric

406 clash between Pac1 and the motor domain to which Pac1 is not bound (Fig. 5A). This

407 strongly suggests that a Pac1-bound dynein would be precluded from adopting the

408 autoinhibited conformation. This also explains the enhanced affinity of Pac1 for the 

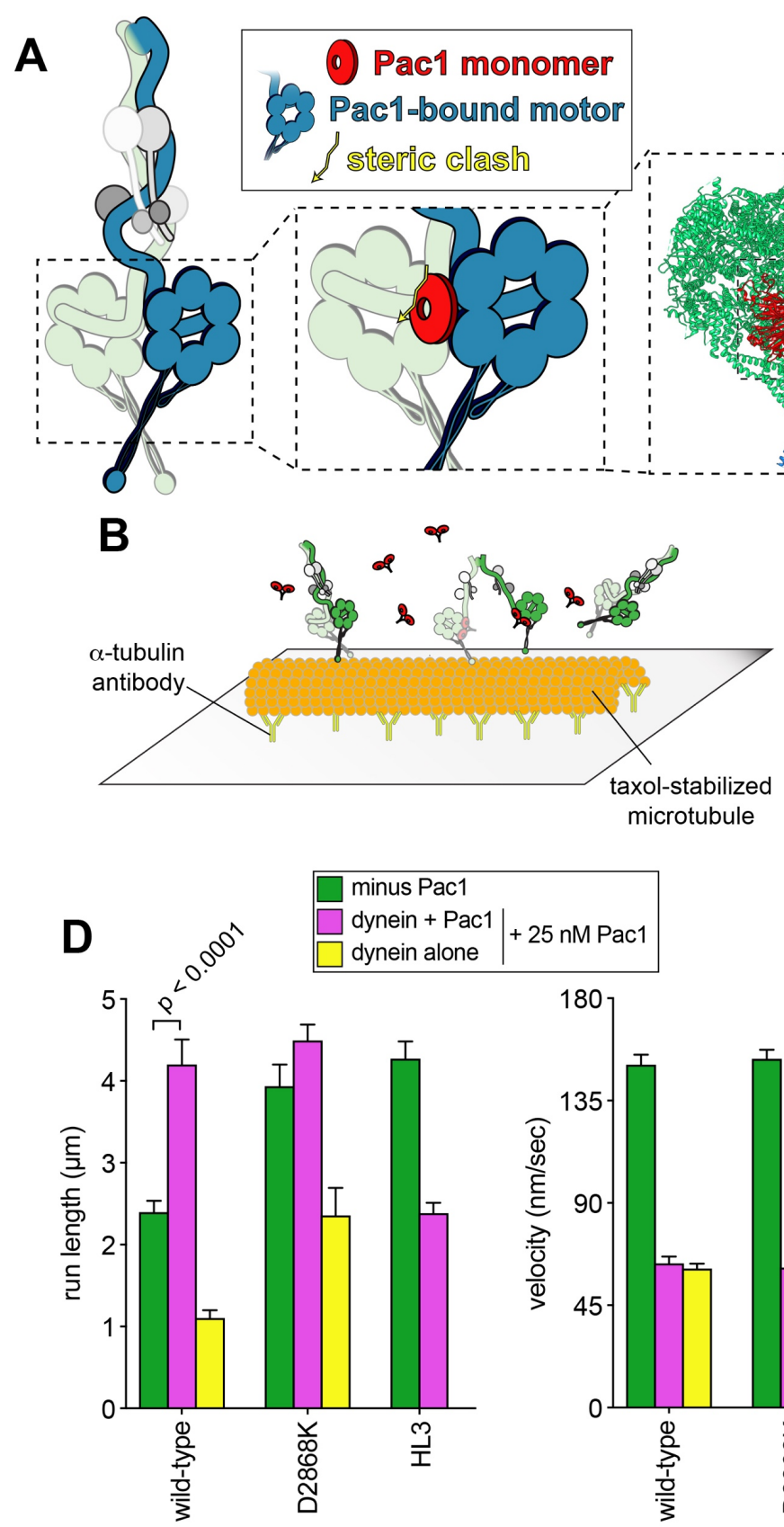
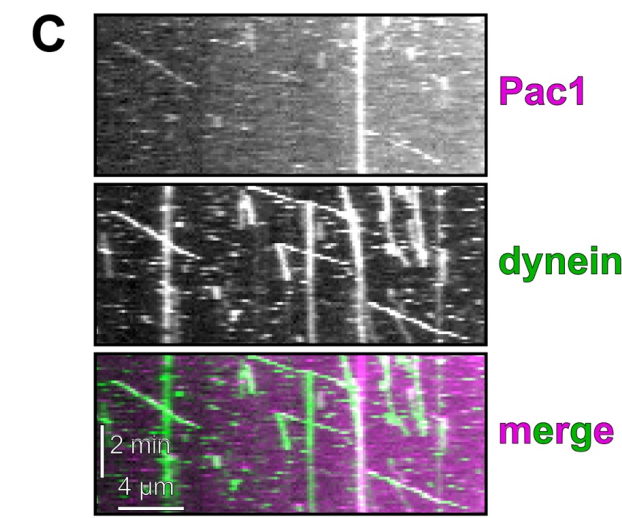

\section{dynein}

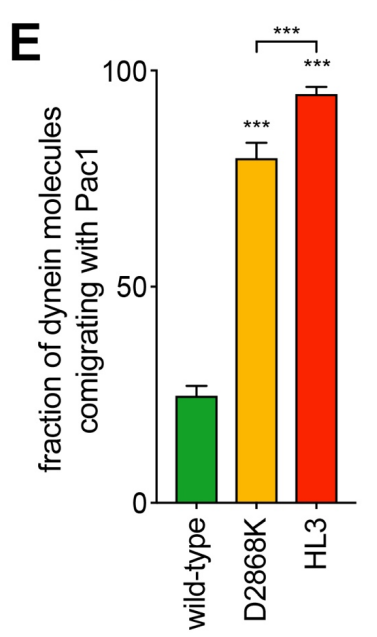

Figure 5. Pac1 promotes release of the autoinhibited conformation of dynein. (A) Cartoon and structural model depicting steric clash between phi particle dynein and Pac1. Structural model was generated by aligning the Pac1-bound dynein monomer structure ( $\mathrm{pdb} 5 \mathrm{VH} 9^{30}$ ) into one of the heavy chains in the phi particle structure (pdb $5 \mathrm{NVU}^{7}$ ). Note the steric clash (depicted with jagged yellow arrow) between the Pac1-bound dynein heavy chain (in blue) with the second heavy chain (in green). (B) Cartoon depicting experimental setup for dynein-Pac1 single molecule assay. (C) Representative kymograph illustrating comigrating dynein-Pac1 complexes in motility buffer supplemented with $150 \mathrm{mM}$ potassium acetate (final concentration). (D) Plots depicting motility parameters (left, run length, from fitting of raw data to one-phase decay; right, velocity) of indicated dyneins moving in the absence (i.e., those not pre-incubated with Pac1, green) or presence of $25 \mathrm{nM}$ Pac1 (dimer concentration). For those experiments in which Pac1 and dynein were pre-incubated, we separately scored those dyneins comigrating with Pac1 (magenta), or migrating without Pac1 (yellow; between 134 - 664 dynein \pm Pac1 from at least two independent experiments were analyzed for each). To acquire movies of dynein alone, 1-second durations were used; however, for two-color dynein + Pac1 movies, we used 3 second durations due to the speed limitations of our microscope hardware. Statistical significance was determined using a Mann-Whitney test. (E) The fraction of dynein molecules migrating with Pac1 is plotted for the indicated dynein. Error bars depict standard error of proportion. Statistical significance was determined by calculating $Z$ scores (unless indicated by brackets, asterisks indicate statistical difference from wild-type; $\left.{ }^{* * *}, \mathrm{p}<0.0001\right)$. 
410 uninhibited dynein conformation, and also for a truncated dynein motor domain

411 fragment (see Fig. 4C).

412 To directly test whether Pac1 could affect the conformational state of dynein, we

413 sought to reassess the effect of Pac1 on dynein motility. In light of our single molecule

414 motility data, we predicted that if Pac1 could promote release of dynein autoinhibition,

415 then it would increase dynein run length. Previous studies describing the effect of Pac1

416 on dynein motility ${ }^{28,29,31}$ directly contrast with recent studies of human LIS1 ${ }^{25-27}$ (the

417 human homolog of Pac1). Specifically, whereas Pac1 was shown to reduce dynein

418 velocity and promote a microtubule-bound state ${ }^{28-30}$, LIS1 was shown to either increase

419 the velocity of human dynein-dynactin complexes ${ }^{25,26}$, or have no effect on velocity ${ }^{27}$. In

420 all these studies, LIS1 was observed comigrating with dynein-dynactin complexes at

421 varying degrees. Thus, to clearly define how Pac1 affects dynein motility, we sought to

422 specifically assess comigrating dynein-Pac1 complexes. However, unlike human LIS125-

$423 \quad 27$, we noted that even at nanomolar concentrations, Pac1 bound extensively along

424 microtubules in our motility buffer (Fig. S4A; with $50 \mathrm{mM}$ potassium acetate). We found

425 that the Pac1-microtubule interaction was sensitive to ionic strength: whereas Pac1

426 strongly bound microtubules in motility buffer supplemented $50 \mathrm{mM}$ potassium acetate,

427 it bound to a much lesser extent in $150 \mathrm{mM}$ potassium acetate (Fig. S4A and B). Thus,

428 we used these latter conditions to assess what effect Pac1 has on dynein motility (Fig.

429 5B).

Two-color imaging of full-length, wild-type dynein preincubated with Pac1 in

431 motility buffer supplemented with $150 \mathrm{mM}$ potassium acetate revealed many instances

432 of their comigration (Fig. 5C and E). From these movies, we separately scored the run 
433 length and velocity values of those dynein molecules that comigrated with Pac1 (Fig.

434 5D, magenta bars, "dynein + Pac1"), and those that moved without any apparent Pac1

435 molecules (yellow bars, "dynein alone"). We noted that comigrating dynein-Pac1

436 complexes moved with significantly longer run lengths than those dynein molecules that

437 were not preincubated with Pac1 (4.2 $\mu \mathrm{m}$ versus $2.4 \mu \mathrm{m} ; \mathrm{p}<0.0001)$. The dynein-Pac1

438 complexes also moved further than those dynein molecules that were not observed

439 comigrating with Pac1 in the same imaging chamber (4.2 $\mu \mathrm{m}$ versus $1.1 \mu \mathrm{m})$. Thus,

440 Pac1 indeed promotes an uninhibited conformational state of motile dynein complexes.

441 If Pac1 increases run lengths of dynein as a consequence of it promoting an

442 uninhibited state, then we reasoned that Pac1 would not do the same to uninhibited

443 dynein mutants. Consistent with the enhanced affinity of Pac1 for these mutants (Fig.

444 4C), we observed a much greater frequency of Pac1 molecules comigrating with

445 dynein ${ }^{\mathrm{D} 2868 \mathrm{~K}}$ and dynein ${ }^{\mathrm{HL} 3}$ (Fig. 5E). However, we noted no Pac1-dependent increase

446 in run length for either of these mutants, further supporting the notion that are already

447 uninhibited. Note that the mean run length value for wild-type dynein with Pac1 (4.2 $\mu \mathrm{m})$

448 is almost identical to that for dynein ${ }^{\mathrm{D} 2868 \mathrm{~K}}$ with Pac1 $(4.5 \mu \mathrm{m} ; \mathrm{p}=0.6187)$, and dynein ${ }^{\mathrm{HL} 3}$

449 alone $(4.3 \mu \mathrm{m} ; \mathrm{p}=0.0620)$, indicating these all represent similar degrees of uninhibited

450 conformational states. As a side note, consistent with the labile nature of the phi particle

451 conformation of the overexpressed wild-type dynein complex, we noted that the extent

452 of its colocalization with Pac1 increased substantially over time (Fig. S2C and D). Taken

453 together, these data indicate that Pac1 stabilizes the uninhibited conformational state of

454 motile dynein complexes. 
Microtubule-bound Pac1 but not dynein-bound Pac1 reduces dynein velocity

$$
\text { Given the wealth of information pertaining to the Pac1-dependent velocity }
$$
reduction of dynein $28,29,31$, we sought to further address the underlying basis for this

461 phenomenon. Given its propensity to bind microtubules in our motility buffer, we

462 hypothesized that the Pac1-dependent reduction in dynein velocity is a direct

463 consequence of its ability to bind microtubules (in a manner analogous to dynein

464 velocity reduction by the microtubule-associated protein, She $\left.{ }^{54,55}\right)$. Consistent with this

465 possibility, structural analysis revealed that Pac1 contacts dynein at a region that is

466 proximal to the microtubule surface $(\leq 6.8 \mathrm{~nm}$; Fig. S5A and B; this does not take into

467 account the unstructured E-hooks, which extend away from the surface). Our first clue

468 that this may be the case came from separately analyzing dynein complexes that

469 comigrated with Pac1 versus those that did not (Fig. 5D, yellow versus magenta bars).

470 Given the low, but detectable prevalence of Pac1 along microtubules in these conditions

471 (see Fig. S4A and B), motile dynein complexes still encounter microtubule-bound Pac1

472 during a processive run. We found that those dynein complexes that comigrated with

473 Pac1 moved with a very similar reduction in velocity as those that did not (Fig. 5D; $p=$

474 0.5093). Notably, those dynein complexes that did not comigrate with Pac1 exhibited

475 run lengths somewhat lower that dynein molecules that moved in the absence of Pac1

476 (compare green and yellow bars). Thus, dynein velocity reduction occurs in a manner

477 that is independent of being bound to Pac1 during a processive run, while processivity

478 enhancement occurs in a manner that requires a stable interaction with Pac1. 
bioRxiv preprint doi: https://doi.org/10.1101/684290; this version posted June 27, 2019. The copyright holder for this preprint (which was not certified by peer review) is the author/funder. All rights reserved. No reuse allowed without permission.

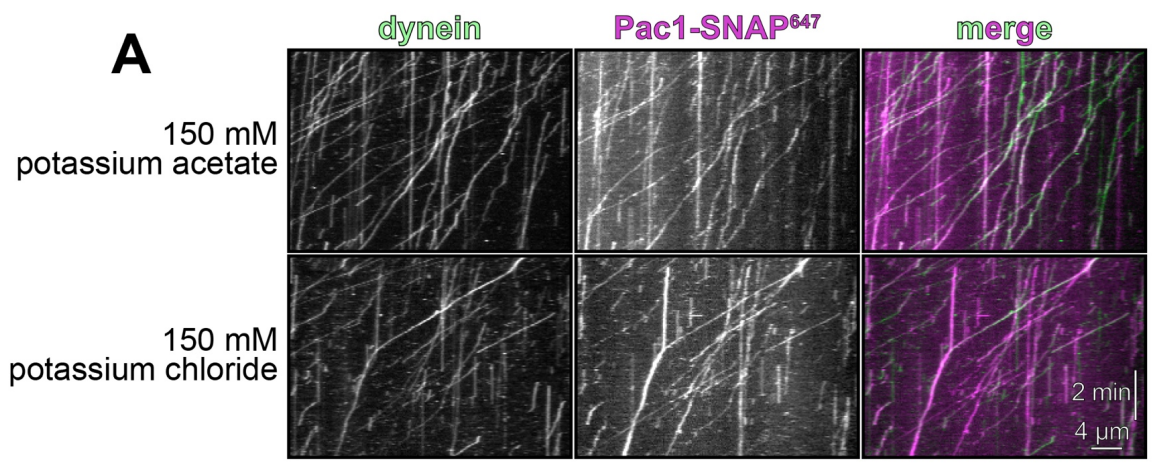

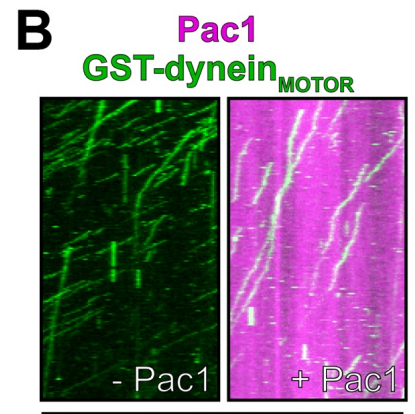

$50 \mathrm{mM}$ potassium acetate
C

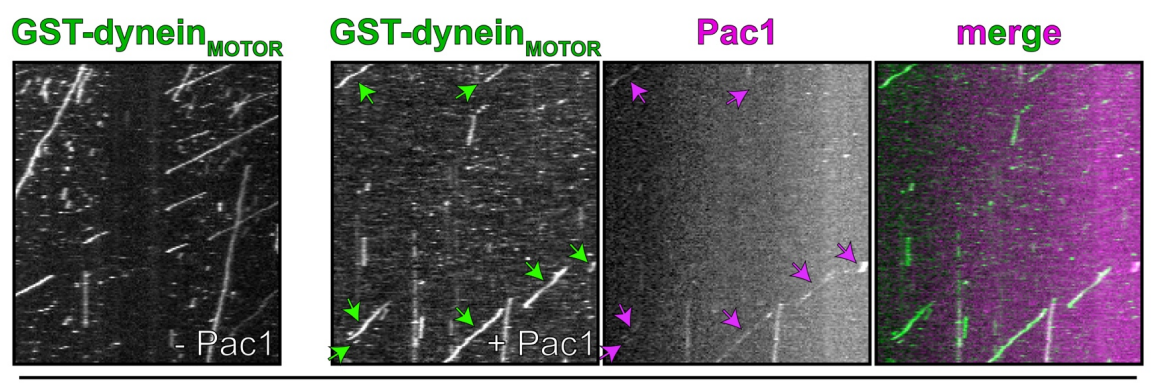

$150 \mathrm{mM}$ potassium chloride
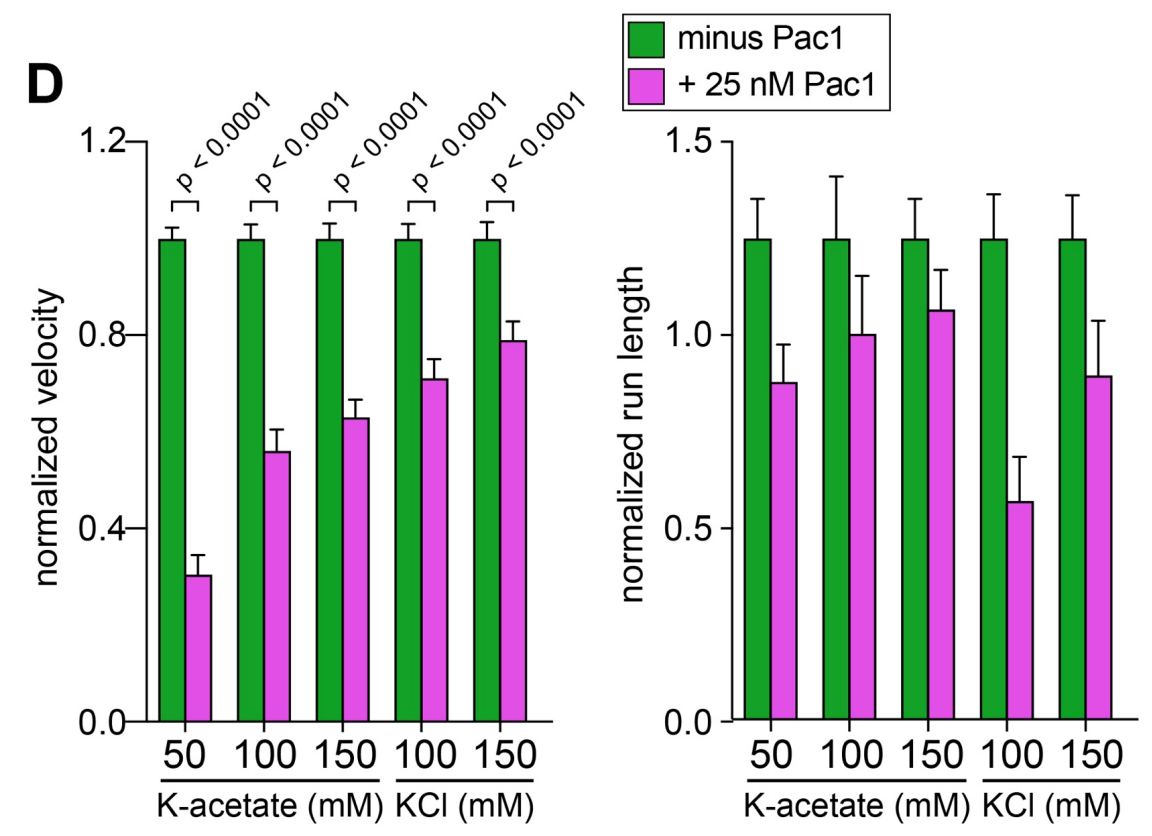

E

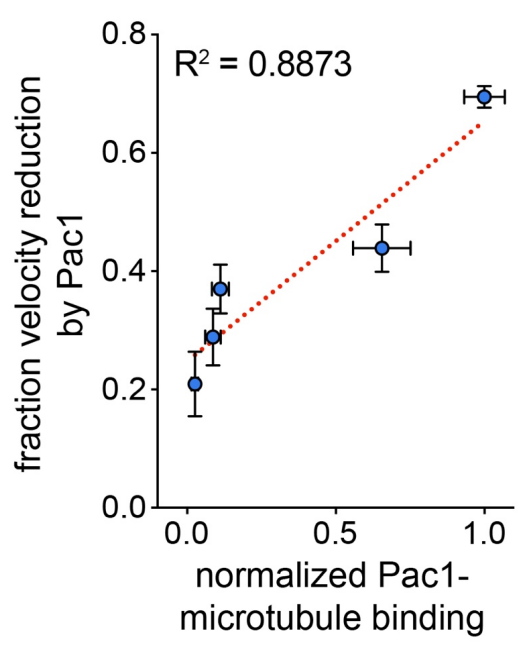

Figure 6. Reducing Pac1-microtubule binding minimizes Pac1-mediated dynein velocity reduction. (A) Representative kymographs depicting dynein ${ }^{\mathrm{D} 286 \mathrm{~K}}$ and Pac1 comigrating in single molecule assay in buffers with increasing ionic strength, as indicated. Note that Pac1 and dynein still interact robustly in this assay in both buffer conditions (as apparent by a high degree of colocalization). (B and C) Representative kymographs depicting different motility characteristics of GST-dynein ${ }_{\text {MOTOR }}$ in the presence of Pac1 when the latter is either extensively bound to the microtubule (B, in buffer supplemented with $50 \mathrm{mM}$ potassium acetate), or to a much less extent (C, in buffer supplemented with $150 \mathrm{mM}$ potassium chloride). (D) Plots depicting normalized motility parameters (left, normalized run length, from fitting of raw data to one-phase decay; right, normalized velocity) of GST-dynein ${ }_{\text {MOTOR }}$ moving in the absence (green) or presence (magenta) of $25 \mathrm{nM}$ Pac1 (dimer concentration; between 226 - 396 motors from two independent experiments were analyzed for each point). Error bars indicate standard error. (E) Plot depicting the relative degree of microtubule binding (normalized to 1; see Fig. S4B) versus the fraction velocity reduction of GST-dynein ${ }_{\text {MOTOR }}$ by Pac1. The points (with error bars representing standard error) were fit to a linear regression that indicates a strong correlation between degree of Pac1-microtubule binding and Pac1-mediated dynein velocity reduction. 
To further investigate the effect of Pac1 on dynein velocity, we sought to establish conditions in which Pac1-microtubule binding was further minimized. We

483 found that motility buffer supplemented with $150 \mathrm{mM}$ potassium chloride resulted in a 484 much lower, although still somewhat detectable degree of Pac1-microtubule binding 485 (Fig. S4A and B). Given the sensitivity of dynein microtubule binding and motility to high 486 salt buffers ${ }^{56}$, we chose these conditions as an upper limit for ionic strength for our 487 motility buffer. Importantly, when compared to buffer supplemented with $150 \mathrm{mM}$ 488 potassium acetate, buffer with $150 \mathrm{mM}$ potassium chloride did not appear to negatively 489 impact the Pac1-dynein interaction, as assessed from two-color imaging of dynein ${ }^{\mathrm{D} 2868 \mathrm{~K}}$ and Pac1 (Fig. 6A).

We next sought to correlate the degree of Pac1-mediated dynein velocity

492 reduction to the extent of microtubule binding by Pac1. We used the GST-dyneinmotoR

493 fragment which has been used extensively in prior Pac1 studies, and exhibits a strong

494 velocity reduction phenotype in low ionic strength buffers ${ }^{28-30}$. As previously reported, 25

$495 \mathrm{nM}$ Pac1 led to a strong (69.5\%) reduction in velocity in the low ionic strength buffer (50 $496 \mathrm{mM}$ potassium acetate; Fig. 6B and D, and Fig. S5C and D), in which Pac1 extensively 497 binds along the microtubule lattice (Fig. S4A and B). However, as ionic strength was 498 increased with either potassium acetate or potassium chloride, we noted the effect of 499 Pac1 on GST-dynein мотоR velocity was substantially reduced (Fig. 6C and D, and Fig. 500 S4D, S5C and D). We plotted the degree of velocity reduction by Pac1 (as shown in Fig. 501 6D) against the extent of Pac1-microtubule binding in each condition (as shown in Fig. 502 S4B). Fitting these points to a linear regression revealed a very strong correlation 503 between microtubule binding by Pac1 and its ability to affect dynein velocity (Fig. 6E; $R^{2}$ 
$504=0.8873)$. Thus, in contrast to processivity enhancement by Pac1 - which occurs in a

505 manner that is independent of Pac1-microtubule binding - velocity reduction of dynein

506 by Pac1 appears to occur only when Pac1 is bound to microtubules. Taken together,

507 these findings support a model in which Pac1 is not in fact an inhibitor, but rather an

508 activator of dynein (see Discussion).

\section{DISCUSSION}

In summary, we have shown that like its human orthologue, yeast cytoplasmic

513 dynein adopts an autoinhibited conformation. Furthermore, we have identified a clear

514 biological relevance for this autoinhibited state. Specifically, the phi particle

515 conformation plays a role in coordinating dynein localization and activity within the cell.

516 This becomes abundantly clear in cells expressing uninhibited dynein mutants, which

517 localize to microtubule plus ends and the cell cortex to a greater extent (the former due

518 to an enhanced affinity for Pac1, and the latter due to increased dynactin binding).

519 Moreover, our work identifies a novel role for Pac1 in regulating dynein autoinhibition.

520 Our biochemical, single molecule, genetic, and cell biological data supported by

521 structural analysis reveals the mechanism by which Pac1 modulates the equilibrium

522 between the inhibited and uninhibited states of dynein (see below). Finally, our findings

523 reveal that prior studies describing the role of Pac1 in effecting dynein velocity reduction

524 are for the most part, if not entirely, a consequence of Pac1's ability to bind

525 microtubules in low ionic strength buffers.

Based on our findings, we propose the following model for dynein function (see

527 Figure 7): (1) Dynein stochastically switches between the inhibited and uninhibited

528 conformational states. (2) Pac1 binds to dynein when it is in the uninhibited state, which 


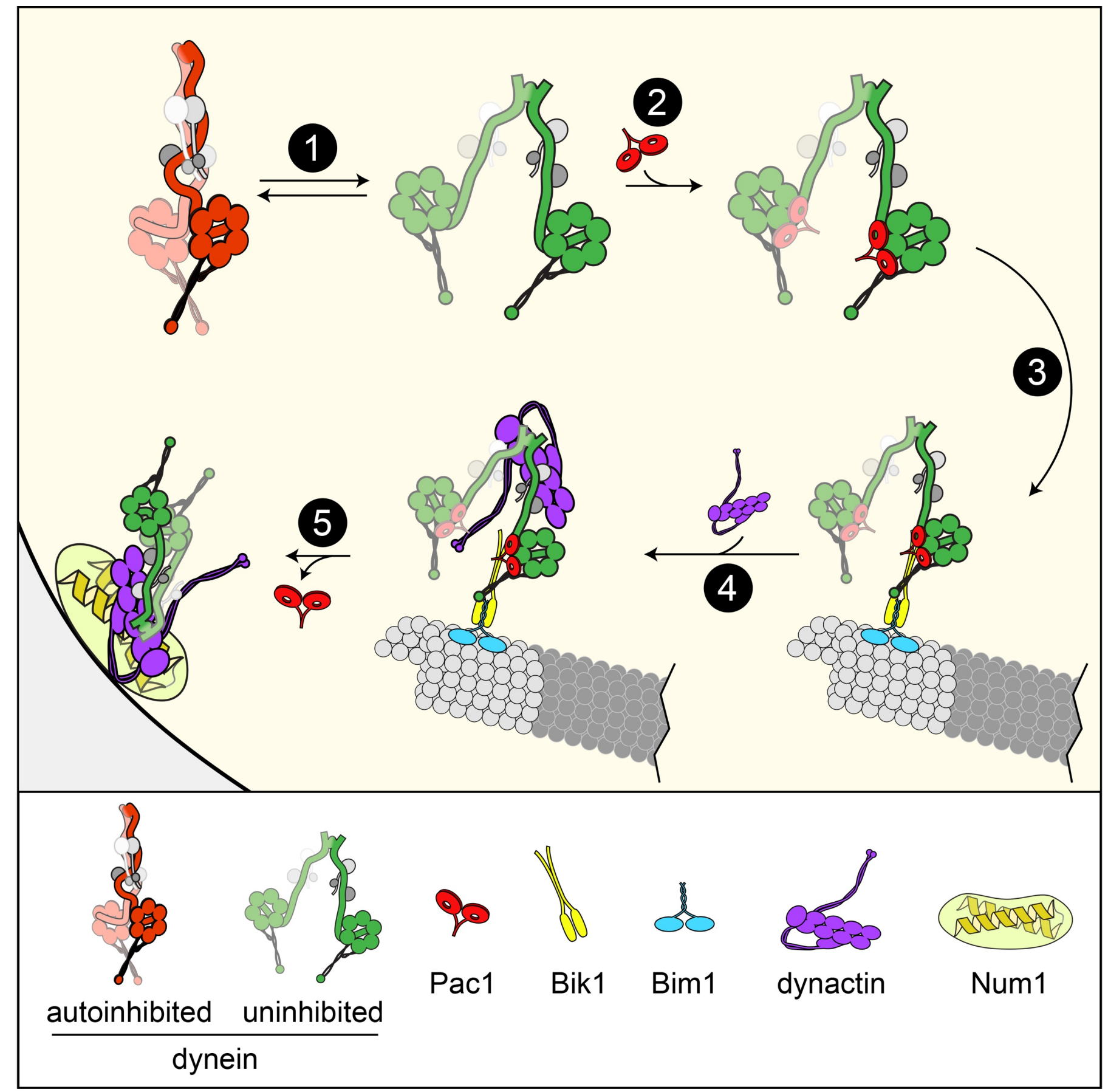

Figure 7. Model for dynein and Pac1 activity in cells. Our data support a model whereby dynein stochastically switches between open and closed states (step 1), the former of which is stabilized by Pac1 binding (step 2). The dynein-Pac1 complex associates with microtubule plus ends via direct interactions with Bik1 (step 3), which associates with plus ends by an unknown mechanism that may rely partly on Bim1. The plus end dynein-Pac1 complex associates with dynactin (step 4), which is then offloaded to cortical Num1 receptor sites (step 5). Given the lack of apparent Pac1 cortical foci, Pac1 likely dissociates either concomitant with, or subsequent to dynein-dynactin offloading. 
530 consequently prevents dynein from switching to the autoinhibited conformation. (3) The

531 Pac1-dynein complex associates with microtubule plus ends ${ }^{46}$ due to their affinity for

532 Bik1 ${ }^{57}$ (homolog of human CLIP-170), which associates with plus ends due in part to its

533 interaction with Bim158 (homolog of human EB1). (4) As a consequence of it being in an

534 uninhibited conformational state ${ }^{7}$, plus end-bound dynein interacts with dynactin. This

535 interaction takes place in the absence of the presumed adaptor molecule, Num $1^{47}$.

536 Although dynein is likely in an open, uninhibited state at microtubule plus ends (due to

537 the requisite presence of $\mathrm{Pac} 1^{59}$ ), the precise configuration of the motor domains of this

538 adaptor-free dynein-dynactin complex (which also occurs with human proteins ${ }^{25,27,60}$ ) is

539 unclear. However, the fact that these complexes do not engage in minus end-directed

540 motility - in either budding yeast ${ }^{47}$, or with reconstituted human proteins ${ }^{25,27,60}$ _

541 suggests that dynein is inactive at this site, despite being uninhibited. This lack of

542 motility could be due in part to its strong affinity for proteins directly bound to the plus

543 end, and/or due to the dynein heads not being appropriately arranged for proper motility

544 (i.e., in a parallel configuration), which has been observed for human dynein in complex

545 with dynactin and the adaptor BicD27 (see below). (5) Upon encountering Num1 at the

546 cell cortex, the dynein-dynactin complex is offloaded ${ }^{31}$ and activated for motility ${ }^{47}$,

547 possibly due to the arrangement of the motor heads in a parallel manner that is

548 conducive for motility ${ }^{7}$. It is interesting to note that the HL3 mutant, which is our most

549 processive motor in single molecule experiments (see Fig. 4B), is completely inactive in

550 cells, as indicated by our cell biological data (see Fig. 4F). This could be a consequence

551 of the helical linker disrupting the adoption of a parallel head configuration that is 
552 potentially needed for cellular dynein-dynactin activity ${ }^{7}$. It also indicates that in vitro

553 single molecule motility is not necessarily a good predictor of cellular activity.

Rather than acting as an inhibitor, our studies indicate that Pac1 in fact promotes

555 cellular dynein activity by stabilizing the uninhibited conformational state (Fig. 7, step 2).

556 Our data show that prior observations of a Pac1-mediated velocity reduction

557 phenotype $28-31$ are a consequence of the low ionic strength buffers used for these

558 assays. Although we still observe a small effect of Pac1 on dynein velocity even at

559 higher ionic strengths (21.0\% reduction), this is likely due to residual microtubule-

560 binding by Pac1 in the highest ionic strength buffer (see Fig. S4A, B and D). These data

561 challenge the current model for Pac1 activity, whereby its binding to the motor domain

562 sterically blocks dynein's mechanochemical cycle ${ }^{29}$. Our data indicate that Pac1 likely

563 reduces dynein velocity in low ionic strength buffers in vitro by simply exerting drag on

564 dynein via simultaneous contacts with dynein and the E-hooks of microtubules (see Fig.

565 S4C and Fig. S5A and B), similar to prior observations with She1 on dynein motility ${ }^{54}$.

566 Given the microtubule-binding-dependent effect of Pac1 on dynein velocity reduction,

567 this raises the question of whether microtubule-binding by Pac1 is a relevant activity in

568 live cells. Several lines of evidence indicate that this is likely not the case. Imaging of

569 Pac1 in live cells reveals it only localizes to microtubule plus ends, and not along the

570 microtubule lattice $44,46,61$. The interaction of Pac1 with microtubule plus ends is indirect

571 (as depicted in Figure 7), as it relies on the presence of dynein ${ }^{46}$ and the CLIP-170

572 homolog, Bik146,61. Finally, previous studies assessing the role of LIS1 in human dynein

573 function have observed no microtubule-binding activity of LIS1 ${ }^{25-27}$. In fact, in contrast to

574 an inhibitory function, two of these studies observed a velocity increase in dynein- 
575 dynactin-BicD2 motility due to LIS125,26. Finally, a previous model describes Pac1

576 inhibiting dynein release from microtubules as the mechanism by which it prevents

577 dynein from walking away from the plus end ${ }^{28-30}$; however, in direct contradiction to this

578 mode of action, the microtubule-binding domain of dynein is dispensable for its

579 accumulation at microtubule plus ends ${ }^{47}$. In summary, we favor the hypothesis that

580 Pac1 is an activator, not an inhibitor of dynein motility. This role is also supported by

581 evidence in which overexpression of Pac1 leads to increased plus end and cortical

582 localization of dynein, and increased cellular dynein activity ${ }^{46}$.

$583 \quad$ Although Pac1 is required for dynein localization and activity in cells expressing

584 wild-type dynein, we show that this need for Pac1 can be bypassed when dynein

585 autoinhibition is prevented by mutagenesis (e.g., K1475E and D2868K). This is also

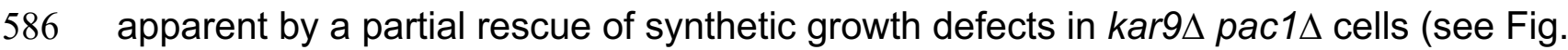

587 4E and Fig. S3A). This is additional support for a role for Pac1 in promoting an

588 uninhibited dynein conformation. It is interesting to note that in spite of the high degree

589 of cortical localization of the uninhibited dynein mutants in pac1 $1 \Delta$ cells, these cells

590 exhibit much fewer dynein-mediated spindle movements than wild-type cells (Fig. 4F).

591 Thus, offloading of dynein to the cell cortex is more conducive to dynein activity than

592 simple recruitment from the cytoplasm. Although the reasons for this are unclear, we

593 propose that the offloading mechanism is optimally suited to maximize cortical dynein

594 activity. Given the large surface area of the cell cortex ( $28 \mu \mathrm{m}^{2}$; assuming a sphere with

595 a $3 \mu$ m diameter) with respect to the small number of diffraction limited cortical dynein

596 foci ( 0-2 foci in the daughter cell; e.g., Fig. 3C), the probability of one of the small

597 number of astral microtubules ( 1-2 in the daughter cell) contacting a cortical dynein- 
598 dynactin complex to initiate a spindle movement into the daughter cell is very low.

599 However, if the microtubule delivers the motor that will subsequently transport it, the

600 reliance on a stochastic search-based process to initiate a spindle movement event is

601 eliminated. Analysis of dynein-mediated spindle movements in budding yeast revealed

602 that approximately $40 \%$ of microtubule-cortex interactions lead to dynein-mediated

603 spindle movements ${ }^{5}$, much greater than would be expected if the cell relied on a

604 stochastic search-based process. Thus, by preventing direct recruitment from the

605 cytoplasm, and requiring a plus end-mediated delivery mechanism, the phi particle, with

606 support from Pac1, ensures that dynein-mediated spindle movements occur within a

607 reasonable timeframe.

608 In addition to the phi particle restricting localization in cells, we also found that it

609 plays a role in reducing processivity in an in vitro context. Given the high proportion of

610 phi particles observed in our negative stain EM images (52.9\% in phi conformation,

611 versus $20.0 \%$ in an open state; Fig. S1), it is surprising that yeast dynein is processive

612 at all. Our data indicate that yeast dynein stochastically switches from open to closed

613 states in the middle of a processive run. Given the increased processivity of the

614 uninhibited mutants, switching to a closed, inhibited state likely leads to termination of a

615 run, which is likely due to a microtubule release event. This is consistent with the phi

616 particle exhibiting lower affinity for microtubules than the open, uninhibited state ${ }^{7}$. Our

617 findings also raise interesting questions regarding the distinct motility capabilities of

618 yeast versus human dynein, the latter of which requires dynactin and an adaptor for

619 processive single molecule motility ${ }^{1,2}$. In particular, why is yeast dynein processive

620 without such factors, while human dynein is not? Cryo-EM data of human dynein in the 
621 absence and presence of dynactin revealed that dynactin binding orients the motor

622 domains in a parallel configuration, suggesting this is the key to dynactin-triggered

623 processive motility ${ }^{7}$. It is possible that the motor domains of yeast dynein have a higher

624 propensity to adopt a parallel configuration in the absence of dynactin binding; however,

625 higher resolution structural data will be required to determine if this is indeed the case.

626 Although our work demonstrates a role for the phi particle in budding yeast

627 dynein function, several lines of evidence indicate its importance in humans. For

628 instance, a previous study showed that mutations that disrupt the human dynein phi

629 particle lead to defects in dynein localization and function in human cells ${ }^{7}$. Similar to our

630 observation that yeast dynein mutants localize to the SPBs to a greater extent, human

631 dynein mutants localize more extensively to the spindle poles ${ }^{7}$. As further evidence for

632 the importance of the autoinhibited conformation, at least three different mutations that

633 map to the phi particle contact surfaces have been identified in patients suffering from

634 neurological disease (i.e., malformations in cortical development): E1518K, R1567Q,

635 and R1603T ${ }^{62-64}$ (equivalent to E1425, K1475, and K1510; see residues with "*” in Fig.

636 2A). We previously showed that a K1475Q dynein mutant exhibits phenotypes much

637 like those described for K1475E (i.e., increased single molecule run lengths and cortical

638 localization), and leads to compromised dynein function in cells ${ }^{34}$. Thus, the phi particle

639 is an important, highly conserved conformational state that is used by organisms

640 throughout evolution to ensure appropriate dynein activity.

\section{AUTHOR CONTRIBUTIONS}

643 S.M.M. and M.G.M. designed the study. M.G.M. performed the bulk of the in vitro and 644 cellular assays, with some support from S.M.M. and J.M.G. The in vitro and cellular data 
645 were analyzed by M.G.M. Negative staining, grid preparation and electron microscopy

646 was performed by Garry P. Morgan at the University of Colorado Boulder Electron

647 Microscopy facility. Single particle analysis was performed by S.M.M. with assistance

648 from the EM facility support staff. Plasmids were generated by S.M.M. while yeast

649 strains were generated by M.G.M. and J.M.G. The manuscript was written by S.M.M.

650 with assistance from M.G.M.

\section{ACKNOWLEDGEMENTS}

653 We are grateful to Samara Reck-Peterson for the 8His-ZZ-Pac1-SNAP expressing

654 yeast strain, and members of the Markus and DeLuca laboratories for valuable

655 discussions. Electron microscopy was done at the University of Colorado, Boulder EM

656 Services Core Facility in the MCDB Department, with the technical assistance of facility

657 staff. This work utilized the RMACC Summit supercomputer, which is supported by the

658 National Science Foundation (awards ACl-1532235 and ACl-1532236), the University of

659 Colorado Boulder and Colorado State University. The RMACC Summit supercomputer

660 is a joint effort of the University of Colorado Boulder and Colorado State University. We

661 are also extremely grateful to Erin Osborne-Nishimura, David King, and Samuel

662 Bowerman for their invaluable assistance with using software on Summit. This work was

663 funded by the NIH/NIGMS (GM118492 to S.M.M.).

664

665 METHODS

666 Media and strain construction 
Strains are derived from either W303 or YEF473A 65 and are available upon

668

669

670

671

672

673

674

675

676

677

678

679

680

681

682

683

684

685

686

687

688

689

request. We transformed yeast strains using the lithium acetate method ${ }^{66}$. Strains carrying mutations were constructed by PCR product-mediated transformation ${ }^{67}$ or by mating followed by tetrad dissection. Proper tagging and mutagenesis was confirmed by PCR, and in most cases sequencing (all point mutations were confirmed via sequencing). Fluorescent tubulin-expressing yeast strains were generated using plasmids and strategies described previously ${ }^{68,69}$. Strains overexpressing the yeast dynein complex were generated by transforming p8His-ZZ-SNAPf-Dynein or p8His-ZZHALO-Dynein (wild-type or mutants) linearized by digestion with Apal (cuts within the URA3 gene; see Fig. 1A). Integration was confirmed by PCR. Yeast synthetic defined (SD) media was obtained from Sunrise Science Products (San Diego, CA).

\section{Plasmid generation}

For overexpression and purification of the yeast dynein complex (wild-type or mutants), we generated a polycistronic plasmid expressing all four dynein complex subunits using strategies analogous to the biGBAC assembly ${ }^{70}$. We first made a yeast expression "library" vector - pLIBy - which enables generation of a gene expression cassettes (GEC) with a strong, inducible GAL1 promoter (GAL1p) on the 5' end, and a synthetic terminator sequence $\left(\mathrm{T}_{\text {synth }}{ }^{37}\right)$ on the 3 ' end. A PCR product encompassing GAL1p, and an oligonucleotide containing $\mathrm{T}_{\text {synth } 3}{ }^{37}$ and a multicloning site (Xbal-NotlSpel-BamHI) were assembled into pRS305 digested with BamHI and Notl using Gibson assembly ${ }^{71}$, yielding pLIBy. We also generated a yeast genomic-integration vector with optimized linker sequences for Gibson assembly ${ }^{70}$ flanked by Pmel restriction sites 
690 (equivalent to pbiG1a and pbiG1b). These plasmids - pbiG1ay and pbiG1by- were

691 generated by using Gibson assembly to insert a PCR product encompassing these

692 elements from pbiG1a and pbiG1 $b^{70}$ into pRS306. PCR products encompassing the

693 DYN2 (without the native intron), DYN3 or PAC11 open reading frames were

694 assembled into pLIBy digested with BamHI and Notl. Subsequently, these GECs were

695 amplified from each respective pLIBy vector using oligonucleotides that include regions

696 for priming preceded on the 5' end by predefined "Cas" sequences" 70 : the DYN2 GEC

697 was amplified with Cas $\alpha$-forward and Cas $\beta$-reverse; the DYN3 GEC was amplified with

698 Cas $\beta$-forward and Cas $\gamma$-reverse; and, the PAC11 GEC was amplified with Cas $\gamma$-forward

699 and Cas $\omega$-reverse. These three PCR products were assembled into pbiG1by digested

700 with Swal to generate pbiG1by:GAL1p:Dyn2::GAL1p:DYN3::GAL1p:PAC11.

701 We generated pLIBy:6His-Strep/I-SNAPf-DYN1 using Gibson assembly.

702 However, due to complications generating a PCR product from this vector, we chose to

703 clone everything into this vector. We first substituted the LEU2 expression cassette in

704 the pLIBy backbone with a URA3 marker by assembling a PCR product encompassing

705 the URA3 cassette from pRS306 into pLIBy:6His-Strep/I-SNAPf-DYN1 digested with

706 Kasl and AatlI, yielding pLIBy:6His-StrepII-SNAPf-DYN1::URA3. To enable assembly of

707 the DYN2/DYN3/PAC11 polygene cassette into pLIBy:6His-Strep/I-SNAPf-

708 DYN1::URA3, we inserted the optimized "B" and "C" linker sequences for Gibson

709 assembly ${ }^{70}$ into this plasmid by assembling a PCR product encompassing "B"-Pmel

710 site-"C" into pLIBy:6His-Strep/I-SNAPf-DYN1::URA3 digested with Kpnl and Sall.

711 Subsequent to digestion with Pmel, this plasmid was assembled with the Pmel

712 restriction digest product from pbiG1by:GAL1p:Dyn2::GAL1p:DYN3::GAL1p:PAC11 
713 (encompassing GAL1p:Dyn2::GAL1p:DYN3::GAL1p:PAC11), yielding pLIBy:

714 GAL1p:Dyn2::GAL1p:DYN3::GAL1p:PAC11::GAL1p:6His-StrepII-SNAPf-Dyn1::URA3,

715 hereafter referred to as p6His-Strepll-SNAPf-Dynein. Prior to using this plasmid for pilot

716 tests, we decided to swap the 6His-Strepll affinity tag for an $8 \mathrm{His}-\mathrm{ZZ}$ tag (followed by a

717 tandem TEV protease recognition site). We did this by assembling a PCR product

718 encompassing 8His-ZZ into p6His-Strepll-SNAPf-Dynein digested with Aatll and Xhol,

719 yielding p8His-ZZ-SNAPf-Dynein. We replaced the SNAPf tag with a HALO tag using a

720 similar strategy, yielding p8His-ZZ-HALO-Dynein. All mutations were engineered into

721 these plasmids using common strategies.

723 Protein purification

724 Purification of Pac1-FLAG-SNAP was performed as previously described ${ }^{28}$.

725 Purification of yeast dynein (ZZ-TEV-Dyn1-HALO, under the native DYN1 promoter; or,

726 ZZ-TEV-HALO-(or SNAPf)-Dynein, with all genes under control of the GAL1p promoter;

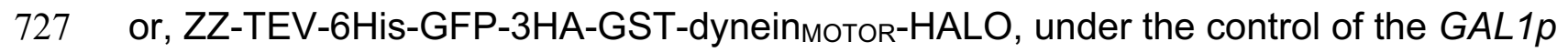

728 promoter) was performed as previously described with minor modifications used for the

729 overexpressed complex ${ }^{28,54}$. Briefly, yeast cultures were grown in YPA supplemented

730 with either $2 \%$ glucose (for non-overexpressed full-length dynein) or $2 \%$ galactose (for

731 the GAL1p-inducible strains), harvested, washed with cold water, and then resuspended

732 in a small volume of water. The resuspended cell pellet was drop frozen into liquid

733 nitrogen and then lysed in a coffee grinder (Hamilton Beach). For most purifications

734 (with exception of those used for negative stain/EM imaging) we used the following

735 procedure: after lysis, 0.25 volume of $4 \mathrm{X}$ dynein lysis buffer (1X buffer: $30 \mathrm{mM}$ HEPES, 
$736 \mathrm{pH}$ 7.2, $50 \mathrm{mM}$ potassium acetate, $2 \mathrm{mM}$ magnesium acetate, 0.2 mM EGTA)

737 supplemented with $1 \mathrm{mM}$ DTT, $0.1 \mathrm{mM}$ Mg-ATP, $0.5 \mathrm{mM}$ Pefabloc SC (concentrations

738 for $1 \mathrm{X}$ buffer) was added, and the lysate was clarified at $22,000 \times \mathrm{g}$ for $20 \mathrm{~min}$. The

739 supernatant was then bound to IgG sepharose 6 fast flow resin (GE) for 1-1.5 hours at

$740 \quad 4^{\circ} \mathrm{C}$, which was subsequently washed three times in $5 \mathrm{ml}$ lysis buffer, and twice in TEV

741 buffer (50 mM Tris, $\mathrm{pH}$ 8.0, $150 \mathrm{mM}$ potassium acetate, $2 \mathrm{mM}$ magnesium acetate, 1

742 mM EGTA, 0.005\% Triton X-100, 10\% glycerol, 1 mM DTT, 0.1 mM Mg-ATP, 0.5 mM

743 Pefabloc SC). To fluorescently label the motors for single molecule analyses, the bead-

744 bound protein was incubated with either 6.7 $\mu \mathrm{M}$ HaloTag-AlexaFluor660 or HaloTag-

745 TMR (Promega), or SNAP-Surface Alex Fluor 647 (NEB), as appropriate, for 10-20

746 minutes at room temperature. The resin was then washed four more times in TEV

747 digest buffer, then incubated in TEV buffer supplemented with TEV protease for 1-1.5

748 hours at $16^{\circ} \mathrm{C}$. Following TEV digest, the beads were pelleted, and the resulting

749 supernatant was aliquoted, flash frozen in liquid nitrogen, and stored at $-80^{\circ} \mathrm{C}$. Protein

750 preparations used for negative stain/EM imaging were subject to tandem affinity

751 purification. To do so, subsequent to lysis, 0.25 volume of 4 X NiNTA dynein lysis buffer

752 (1X buffer: 30 mM HEPES, pH 7.2, 200 mM potassium acetate, 2 mM magnesium

753 acetate, $10 \%$ glycerol) supplemented with $1 \mathrm{mM}$ beta-mercaptoethanol, $0.1 \mathrm{mM} \mathrm{Mg-}$

754 ATP, $0.5 \mathrm{mM}$ Pefabloc SC (concentrations for $1 \mathrm{X}$ buffer) was added, and the lysate was

755 clarified as above. The supernatant was then bound to NiNTA agarose for 1 hour at

$7564^{\circ} \mathrm{C}$, which was subsequently washed three times in $5 \mathrm{ml}$ NiNTA lysis buffer. The

757 protein was eluted in NiNTA lysis buffer supplemented with $250 \mathrm{mM}$ imidazole by

758 incubation for 10 minutes on ice. The eluate was then diluted with an equal volume of 
dynein lysis buffer, which was then incubated with IgG sepharose 6 fast flow resin for 1

760 hour at $4^{\circ} \mathrm{C}$. The beads were washed and the protein was eluted as described above.

761 Eluted protein was either applied to a size exclusion resin (Superose 6; GE), or snap

762 frozen. The gel filtration resin was equilibrated in GF150 buffer (25 mM HEPES pH 7.4,

$763150 \mathrm{mM} \mathrm{KCl}, 1 \mathrm{mM} \mathrm{MgCl}$, 5 mM DTT, $0.1 \mathrm{mM} \mathrm{Mg-ATP)} \mathrm{using} \mathrm{an} \mathrm{AKTA} \mathrm{Pure.} \mathrm{Peak}$

764 fractions (determined by UV $260 \mathrm{~nm}$ absorbance and SDS-PAGE) were pooled,

765 concentrated, aliquoted, flash frozen, then stored at $-80^{\circ} \mathrm{C}$.

For comparison of elution profiles between yeast and human dynein complexes,

767 the human dynein complex was expressed and purified from insect cells (ExpiSf9 cells;

768 Life Technologies) as previously described with minor modifications ${ }^{1,7}$. Briefly, $4 \mathrm{ml}$ of

769 ExpiSf9 cells at $2.5 \times 10^{6}$ cells/ml, which were maintained in ExpiSf CD Medium (Life

770 Technologies), were transfected with $1 \mu \mathrm{g}$ of bacmid DNA (see above) using

771 ExpiFectamine (Life Technologies) according to the manufacturer's instructions. 5 days

772 following transfection, the cells were pelleted, and $1 \mathrm{ml}$ of the resulting supernatant (P1)

773 was used to infect $300 \mathrm{ml}$ of ExpiSf9 cells $\left(5 \times 10^{6}\right.$ cells $\left./ \mathrm{ml}\right) .72$ hours later, the cells

774 were harvested (2000 x g, $20 \mathrm{~min})$, washed with phosphate buffered saline $(\mathrm{pH} 7.2)$,

775 pelleted again $(1810 \times \mathrm{g}, 20 \mathrm{~min})$, and resuspended in an equal volume of human

776 dynein lysis buffer (50 mM HEPES, pH 7.4, 100 mM NaCl, 10\% glycerol, 1 mM DTT,

$7770.1 \mathrm{mM}$ Mg-ATP, $1 \mathrm{mM}$ PMSF). The resulting cell suspension was drop frozen in liquid

778 nitrogen and stored at $-80^{\circ} \mathrm{C}$. For protein purification, $30 \mathrm{ml}$ of additional human dynein

779 lysis buffer supplemented with cOmplete protease inhibitor cocktail (Roche) was added

780 to the frozen cell pellet, which was then rapidly thawed in a $37^{\circ} \mathrm{C}$ water bath prior to

781 incubation on ice. Cells were lysed in a dounce-type tissue grinder (Wheaton) using 
$782 \geq 150$ strokes (lysis was monitored by microscopy). Subsequent to clarification at

$78322,000 \times \mathrm{g}, 45 \mathrm{~min}$, the supernatant was applied to $2 \mathrm{ml}$ of IgG sepharose fast flow resin

784 pre-equilibrated in human dynein lysis buffer, and incubated at $4^{\circ} \mathrm{C}$ for $2-4$ hours. Beads

785 were then washed with $50 \mathrm{ml}$ of human dynein lysis buffer, and $50 \mathrm{ml}$ of human dynein

786 TEV buffer (50 mM Tris $\mathrm{pH}$ 7.4, $150 \mathrm{mM}$ potassium acetate, $2 \mathrm{mM}$ magnesium acetate,

$7871 \mathrm{mM}$ EGTA, 10\% glycerol, $1 \mathrm{mM}$ DTT, $0.1 \mathrm{mM}$ Mg-ATP). The bead-bound protein was

788 eluted with by incubation with TEV protease overnight at $4^{\circ} \mathrm{C}$. The next morning, the

789 recovered supernatant was applied to a Superose 6 gel filtration column as above.

\section{Single molecule motility assays}

The yeast dynein single-molecule motility assay was performed as previously

793 described with minor modifications ${ }^{54}$. Briefly, flow chambers constructed using slides

794 and plasma cleaned and silanized coverslips attached with double-sided adhesive tape

795 were coated with anti-tubulin antibody $(8 \mu \mathrm{g} / \mathrm{ml}, \mathrm{YL1} / 2$; Accurate Chemical \& Scientific

796 Corporation) then blocked with 1\% Pluronic F-127 (Fisher Scientific). Taxol-stabilized

797 microtubules assembled from unlabeled and fluorescently-labeled porcine tubulin (10:1

798 ratio; Cytoskeleton) were introduced into the chamber. Following a 5-10 minute

799 incubation, the chamber was washed with dynein lysis buffer (see above) supplemented

800 with $20 \mu \mathrm{M}$ taxol. Subsequently, purified dynein motors diluted in motility buffer (30 mM

801 HEPES pH 7.2, 2 mM magnesium acetate, 1 mM EGTA, 1 mM DTT, 1 mM Mg-ATP,

$802 \quad 0.05 \%$ Pluronic F-127, $20 \mu \mathrm{M}$ taxol, and an oxygen-scavenging system consisting of

$8031.5 \%$ glucose, $1 \mathrm{U} / \mathrm{ml}$ glucose oxidase, $125 \mathrm{U} / \mathrm{ml}$ catalase) supplemented with either 50 
$804 \mathrm{mM}$ potassium acetate, or as indicated in figure legend, were introduced in the

805 chamber, and imaged.

806 To image comigrating Pac1-dynein complexes, 500 nM Pac1-SNAP647 (dimer

807 concentration) and $\sim 50$ nM HALO ${ }^{\text {TMR }}$-Dynein were preincubated on ice for 10-15

808 minutes prior to a 20-fold dilution into modified motility buffer (30 mM HEPES pH 7.2, 2

809 mM magnesium acetate, 1 mM EGTA, 1 mM DTT, 1 mM Mg-ATP) supplemented with

810 potassium acetate or potassium chloride as indicated in figure legends, $0.05 \%$ Pluronic

$811 \mathrm{~F}-127,20 \mu \mathrm{M}$ taxol, and an oxygen-scavenging system (as above). The higher yield

812 overexpressed dynein complex was needed for these assays given the low landing rate

813 of dynein in the higher ionic strength buffers. We ensured that comigrating Pac1-

814 SNAP ${ }^{647}$ spots were not due to bleed-through from the HALO ${ }^{T M R}$-dynein channel by

815 performing two-color imaging with $\mathrm{HALO}^{\mathrm{TMR}}$-dynein alone (no spots were apparent in

816 the far-red channel in these cases).

817 TIRFM images were collected using a 1.49 NA 100X TIRF objective on a Nikon

818 Ti-E inverted microscope equipped with a Ti-S-E motorized stage, piezo Z-control

819 (Physik Instrumente), and an iXon X3 DU897 cooled EM-CCD camera (Andor). 488 nm,

$820561 \mathrm{~nm}$, and $640 \mathrm{~nm}$ lasers (Coherent) were used along with a multi-pass quad filter

821 cube set (C-TIRF for 405/488/561/638 nm; Chroma) and emission filters mounted in a

822 filter wheel $(525 / 50 \mathrm{~nm}, 600 / 50 \mathrm{~nm}$ and $700 / 75 \mathrm{~nm}$; Chroma). We acquired images at 1,

8232 , or 3 second intervals for $8-10 \mathrm{~min}$. Velocity and run length values were determined

824 from kymographs generated using the MultipleKymograph plugin for ImageJ

825 (http://www.embl.de/eamnet/html/body kymograph.html). Those motors that moved for

$826 \geq 3$ time points were measured. 


\section{Negative stain electron microscopy and image analysis}

EM grids were prepared with a standard negative stain protocol by applying fresh

830 dynein samples to glow discharged carbon coated 200 mesh copper grids. After $\sim 1$

831 minute incubation, $2 \%$ uranyl acetate was added. 1600 micrographs were collected on a

832 FEI Tecnai F20 200kV TEM equipped with a Gatan US4000 CCD (model 984), at a

833 nominal magnification of $90,000 X$ with the digital pixel size 6.19 angstroms. All image

834 analysis was performed in Relion 3.0 on the University of Colorado Boulder High

835 Performance Computer Cluster, Summit. Particles were manually picked from 20

836 micrographs ( 200 particles), which were used to generate a low resolution 2D class

837 average. Using these 2D averages as a starting point, we then used an iterative

838 process to autopick particles that were used to generate our final 2D averages, and for

839 3D model building (in total, 42,611 particles were used for final averages shown in

840 Figure 1D).

\section{Live cell imaging experiments}

843 For the spindle dynamics assay, cells were arrested with hydroxyurea (HU) for

8442.5 hours, and then mounted on agarose pads containing HU for fluorescence

845 microscopy. Full Z-stacks (23 planes with $0.2 \mu \mathrm{m}$ spacing) of GFP-labeled microtubules

846 (GFP-Tub1) were acquired every 10 seconds for 10 minutes on a stage prewarmed to

$84730^{\circ} \mathrm{C}$. To image dynein localization in live cells, cells were grown to mid-log phase in SD

848 media supplemented with $2 \%$ glucose, and mounted on agarose pads. Images were

849 collected on a Nikon Ti-E microscope equipped with a 1.49 NA 100X TIRF objective, a 
850 Ti-S-E motorized stage, piezo Z-control (Physik Instrumente), an iXon DU888 cooled

851 EM-CCD camera (Andor), a stage-top incubation system (Okolab), and a spinning disc

852 confocal scanner unit (CSUX1; Yokogawa) with an emission filter wheel (ET480/40m for

853 mTurquoise2, ET525/50M for GFP, and ET632/60M for mRuby2; Chroma). Lasers (445

$854 \mathrm{~nm}, 488 \mathrm{~nm}$ and $561 \mathrm{~nm}$ ) housed in a LU-NV laser unit equipped with AOTF control

855 (Nikon) were used to excite mTurquoise2, GFP and mRuby2, respectively. The

856 microscope was controlled with NIS Elements software (Nikon).

858 Statistical analyses

859 Statistical tests were performed as described in the figure legends. T-tests were

860 performed using Graphpad Prism. Z scores were calculated using the following formula:

861

$$
Z=\frac{\left(\hat{p}_{1}-\hat{p}_{2}\right)}{\hat{p}(1-\hat{p})\left(\frac{1}{n_{1}}+\frac{1}{n_{2}}\right)}
$$

862 where:

863

$$
\hat{p}=\frac{y_{1}+y_{2}}{n_{1}+n_{2}}
$$

864 Z scores were converted to two-tailed $\mathrm{P}$ values using an online calculator.

\section{Data availability}

All yeast strains, and datasets generated during and/or analysed during the

868 current study are available from the corresponding author upon request. 


\section{Figure 1. The yeast dynein complex adopts an autoinhibited phi particle}

872 conformation. (A) Schematic of the polycistronic plasmid used to produce the intact

873 yeast dynein complex. Restriction digest with Apal (cuts within URA3 gene) targets the

874 plasmid for homologous recombination into the ura3-1 locus as depicted. (B)

875 Representative kymograph depicting single molecule motility of the purified

876 overexpressed yeast dynein complex. (C) Elution profiles of yeast and human dynein

877 complexes from Superose 6 resin (left), and scans of the same polyacrylamide gel

878 depicting fluorescently labeled Dyn1 (via HaloTag-TMR) and the entire complex (via

879 Sypro Ruby staining; right). (D) Representative negative stain EM class averages of the

880 intact yeast dynein complex. Number of particles used to generate each class indicated

881 in each panel. Classes i - vi depict dynein in the autoinhibited, phi particle conformation,

882 whereas vii $-x$ depict dynein in various open, uninhibited states. (E) 3D models of

883 dynein in the autoinhibited state generated from 2D class averages with (right) and

884 without (left) a high resolution 3D structure of human dynein-1 in the phi particle

885 conformation (pdb 5NVU) manually docked into it. Note that the structures of the two tail

886 domains have been slightly rotated with respect to the motor domains to better fit the 3D

887 model, and that the structures of both TcTEX and Robl have been eliminated due to

888 their absence from the yeast dynein complex. Also see Video S1.

890 Figure 2. Disrupting phi particle contact points extends single molecule run

891 lengths. (A) Cartoon depicting four predicted intermolecular contact surfaces within the

892 motor domains that stabilize the phi particle conformation. Four insets show respective

893 regions of yeast dynein modeled into human dynein phi particle structure. Structural 
894 models were generated using one-to-one threading of the yeast DYN1 sequence into

$8955 \mathrm{NVU}^{7}$ on the Phyre2 server ${ }^{72}$. Residues with magenta asterisks are mutated in patients

896 suffering from neurological disease ${ }^{62-64}$ (see Discussion). (B) Single molecule run length

897 (from fitting of raw data to one-phase decay) and velocity values for wild-type and

898 mutant dyneins with phi particle disrupting mutations (at surfaces 2, 3 and 4, as

899 indicated). Cartoons along vertical axis depict electrostatic interactions (or lack thereof)

900 among residues 1517, 1475 (left circles) and 2868 (right circle) at linker-AAA4 surface.

901 Note that the degree of processivity enhancement is inversely proportional to the

902 number of charge interactions at this surface. Error bars indicate standard error

903 (between 150 - 528 motors from at least two independent experiments were analyzed

904 for each). Statistical significance was determined using a Mann-Whitney test (for run

905 length) or with an unpaired Welch's t test (two-tailed; for velocity; $\left.{ }^{* *}, \mathrm{p} \leq 0.0001\right)$. Also

906 note that we generated and tested the motility of two other point mutants at interface 3,

907 E3441K and R3445D, both of which were inactive in single molecule assays (not

908 shown).

909

910 Figure 3 . The autoinhibited conformation restricts plus end and cortical

911 localization of dynein. (A) Cartoon depicting the two main sites of dynein localization

912 (microtubule plus ends, and cell cortex), and the molecular requirements for each.

913 Dynein plus end localization (1) requires Bik1 $1^{57}$ and $\mathrm{Pac} 1^{44}$, with Bim1 potentially

914 playing some role in this process, but does not require dynactin ${ }^{4}$. Rather, dynactin plus

915 end localization (2) relies on dynein ${ }^{4}$. Subsequent to plus end targeting, dynein-dynactin

916 complexes are offloaded to cortical Num1 sites ${ }^{31,73}$ (3). (B) Plot depicting the frequency 
917 of plus end, SPB (spindle pole body) and cortical targeting for wild-type and mutant

918 Dyn1 ( $\mathrm{n} \geq 32$ mitotic cells for each). Error bars indicate standard error of proportion.

919 Statistical significance was determined by calculating Z scores (see Methods). (C)

920 Representative images of wild-type or mutant dynein (D2868K) localizing in otherwise

921 wild-type or nip100د (dynactin component) cells. Note the lack of cortical localization of

922 Dyn1 $1^{\text {D2868K }}$ in nip1004 cells (white arrowheads, cortical foci; white arrows, plus end foci;

923 blue arrowheads, SPB foci).

925 Figure 4. Release of dynein autoinhibition permits Pac1/LIS1-independent

926 localization and function. (A) Cartoons depicting original, and new models accounting

927 for "unmasking" phenotype observed with Dyn $1^{\mathrm{HL} 3}$ mutant ${ }^{31}$. Wild-type dynein tail

928 domain is unable to associate with Num1 in the absence of plus end-targeting; however,

929 addition of helical linker 3 (HL3) between tail and motor domains permits dynein to

930 associate with Num1 independent of plus end-targeting. Our original model posited that

931 this was a consequence of the motor domain directly precluding the tail domain from

932 contact Num1; however, our new model is that contacts within the motor domain

933 stabilize the phi particle conformation, in which the tail domains are in a twisted state

934 that is unable to interact with Num1. In this latter model, insertion of HL3 prevents the

935 adoption of the phi particle conformation. (B) Single molecule run length (from fitting of

936 raw data to one-phase decay) and velocity values for wild-type and indicated mutant

937 dyneins, as indicated, purified using plasmid-integration strategy described in Figure 1A

938 ( $n \geq 224$ motors for each, from at least two independent experiments; error bars indicate

939 standard error). Statistical significance was determined using a Mann-Whitney test (for 
940 run length) or with an unpaired Welch's t test (two-tailed; for velocity). (C) Bead binding

941 assay illustrating increased affinity of Pac1 for "open” dyneins (dynein ${ }^{\mathrm{D} 2868 \mathrm{~K}}$ and GST-

942 dynein

943 beads, and the bound ("B") and unbound ("U") fractions were resolved by SDS-PAGE.

944 The normalized, relative bound and unbound fractions were determined by measuring

945 band intensities. (D) Plot depicting the fraction of cells with indicated mutant or wild-type

946 Dyn1 foci in pac1s cells ( $\mathrm{n} \geq 34$ mitotic cells for each; "n.o.”, none observed; error bars

947 indicate standard error of proportion). Representative fluorescence images depicting the

948 presence of cortical dynein (Dyn1) and dynactin (Jnm1) in dyn $1^{\text {D2868K }}$ cells (arrowheads,

949 cortical foci; arrows, SPB foci). Statistical significance was determined by calculating Z

950 scores $\left({ }^{* *}, p=0.011 ;{ }^{* *}, p \leq 0.0001\right)$. (E) Serial dilutions of cells with indicated

951 genotype were plated on rich media (YPA supplemented with $2 \%$ glucose) and grown at

$95230^{\circ} \mathrm{C}$ for 2 days (as shown) or 4 days (see Fig. S3A). Note the partial rescue of cell

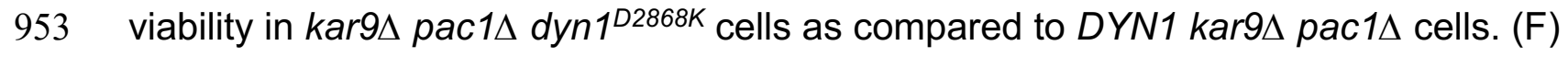

954 Plot depicting number of dynein-mediated spindle movements per cell per minute in

955 hydroxyurea (HU)-arrested cells (all of which are kar9 ; see Methods; $\mathrm{n} \geq 32 \mathrm{HU}$

956 arrested cells for each). (G) Representative time-lapse fluorescence images of a

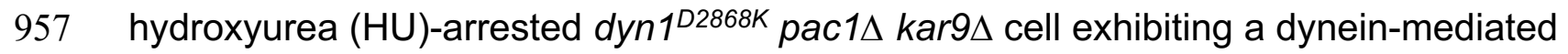

958 spindle movement.

959

960 Figure 5. Pac1 promotes release of the autoinhibited conformation of dynein. (A)

961 Cartoon and structural model depicting steric clash between phi particle dynein and

962 Pac1. Structural model was generated by aligning the Pac1-bound dynein monomer 
963 structure $\left(\mathrm{pdb} 5 \mathrm{VH} 9^{30}\right)$ into one of the heavy chains in the phi particle structure $(\mathrm{pdb}$

$9645 \mathrm{NVU}^{7}$ ). Note the steric clash (depicted with jagged yellow arrow) between the Pac1-

965 bound dynein heavy chain (in blue) with the second heavy chain (in green). (B) Cartoon

966 depicting experimental setup for dynein-Pac1 single molecule assay. (C)

967 Representative kymograph illustrating comigrating dynein-Pac1 complexes in motility

968 buffer supplemented with $150 \mathrm{mM}$ potassium acetate (final concentration). (D) Plots

969 depicting motility parameters (left, run length, from fitting of raw data to one-phase

970 decay; right, velocity) of indicated dyneins moving in the absence (i.e., those not pre-

971 incubated with Pac1, green) or presence of 25 nM Pac1 (dimer concentration). For

972 those experiments in which Pac1 and dynein were pre-incubated, we separately scored

973 those dyneins comigrating with Pac1 (magenta), or migrating without Pac1 (yellow;

974 between 134 - 664 dynein \pm Pac1 from at least two independent experiments were

975 analyzed for each). To acquire movies of dynein alone, 1-second durations were used;

976 however, for two-color dynein + Pac1 movies, we used 3 second durations due to the

977 speed limitations of our microscope hardware. Statistical significance was determined

978 using a Mann-Whitney test. (E) The fraction of dynein molecules migrating with Pac1 is

979 plotted for the indicated dynein. Error bars depict standard error of proportion. Statistical

980 significance was determined by calculating $Z$ scores (unless indicated by brackets,

981 asterisks indicate statistical difference from wild-type; $\left.{ }^{* * *}, p<0.0001 ;{ }^{* *}, p=0.0011\right)$.

983 Figure 6. Reducing Pac1-microtubule binding minimizes Pac1-mediated dynein

984 velocity reduction. (A) Representative kymographs depicting dynein ${ }^{\mathrm{D} 2868 \mathrm{~K}}$ and Pac1

985 comigrating in single molecule assay in buffers with increasing ionic strength, as 
986 indicated. Note that Pac1 and dynein still interact robustly in this assay in both buffer

987 conditions (as apparent by a high degree of colocalization). (B and C) Representative

988 kymographs depicting different motility characteristics of GST-dynein ${ }_{\text {MOTOR }}$ in the

989 presence of Pac1 when the latter is either extensively bound to the microtubule (B, in

990 buffer supplemented with $50 \mathrm{mM}$ potassium acetate), or to a much less extent (C, in

991 buffer supplemented with $150 \mathrm{mM}$ potassium chloride). (D) Plots depicting normalized

992 motility parameters (left, normalized run length, from fitting of raw data to one-phase

993 decay; right, normalized velocity) of GST-dyneinмотоR moving in the absence (green) or

994 presence (magenta) of 25 nM Pac1 (dimer concentration; between 226 - 396 motors

995 from two independent experiments were analyzed for each point). Error bars indicate

996 standard error. (E) Plot depicting the relative degree of microtubule binding (normalized

997 to 1; see Fig. S4B) versus the fraction velocity reduction of GST-dynein

998 The points (with error bars representing standard error) were fit to a linear regression

999 that indicates a strong correlation between degree of Pac1-microtubule binding and

1000 Pac1-mediated dynein velocity reduction.

1001

1002 Figure 7. Model for dynein and Pac1 activity in cells. Our data support a model

1003 whereby dynein stochastically switches between open and closed states (step 1), the

1004 former of which is stabilized by Pac1 binding (step 2). The dynein-Pac1 complex

1005 associates with microtubule plus ends via direct interactions with Bik1 (step 3), which

1006 associates with plus ends by an unknown mechanism that may rely partly on Bim1. The

1007 plus end dynein-Pac1 complex associates with dynactin (step 4), which is then

1008 offloaded to cortical Num1 receptor sites (step 5). Given the lack of apparent Pac1 
cortical foci, Pac1 likely dissociates either concomitant with, or subsequent to dynein-

\section{REFERENCES}

1013

1014

1. Schlager, M. A., Hoang, H. T., Urnavicius, L., Bullock, S. L. \& Carter, A. P. In

1015

1016

1017

1018

1019

1020

1021

1022

1023

1024

1025

1026

1027

1028

1029

1030

1031

1032

1033

1034

1035

1036

1037

1038

1039

1040

1041

1042

1043

1044

1045

1046

1047

1048

1049

1050

1051

1052 vitro reconstitution of a highly processive recombinant human dynein complex. EMBO J 33, 1855-1868, (2014).

2. McKenney, R. J., Huynh, W., Tanenbaum, M. E., Bhabha, G. \& Vale, R. D. Activation of cytoplasmic dynein motility by dynactin-cargo adapter complexes. Science 345, 337-341, (2014).

3. Reck-Peterson, S. L. et al. Single-molecule analysis of dynein processivity and stepping behavior. Cell 126, 335-348, (2006).

4. Moore, J. K., Li, J. \& Cooper, J. A. Dynactin function in mitotic spindle positioning. Traffic 9, 510-527, (2008).

5. Moore, J. K., Sept, D. \& Cooper, J. A. Neurodegeneration mutations in dynactin impair dynein-dependent nuclear migration. Proc Natl Acad Sci U S A 106, 51475152, (2009).

6. Reck-Peterson, S. L., Redwine, W. B., Vale, R. D. \& Carter, A. P. The cytoplasmic dynein transport machinery and its many cargoes. Nat Rev Mol Cell Biol 19, 382-398, (2018).

7. Zhang, K. et al. Cryo-EM Reveals How Human Cytoplasmic Dynein Is Autoinhibited and Activated. Cell 169, 1303-1314 e1318, (2017).

8. Amos, L. A. Brain dynein crossbridges microtubules into bundles. J Cell Sci 93 ( Pt 1), 19-28, (1989).

9. Toropova, K., Mladenov, M. \& Roberts, A. J. Intraflagellar transport dynein is autoinhibited by trapping of its mechanical and track-binding elements. Nat Struct Mol Biol 24, 461-468, (2017).

10. Jordan, M. A., Diener, D. R., Stepanek, L. \& Pigino, G. The cryo-EM structure of intraflagellar transport trains reveals how dynein is inactivated to ensure unidirectional anterograde movement in cilia. Nat Cell Biol 20, 1250-1255, (2018).

11. Torisawa, T. et al. Autoinhibition and cooperative activation mechanisms of cytoplasmic dynein. Nat Cell Biol 16, 1118-1124, (2014).

12. Asante, D., Stevenson, N. L. \& Stephens, D. J. Subunit composition of the human cytoplasmic dynein-2 complex. J Cell Sci 127, 4774-4787, (2014).

13. Kardon, J. R., Reck-Peterson, S. L. \& Vale, R. D. Regulation of the processivity and intracellular localization of Saccharomyces cerevisiae dynein by dynactin. Proc Natl Acad Sci U S A 106, 5669-5674, (2009).

14. Sasaki, S. et al. A LIS1/NUDEL/cytoplasmic dynein heavy chain complex in the developing and adult nervous system. Neuron 28, 681-696, (2000).

15. Wynshaw-Boris, A. \& Gambello, M. J. LIS1 and dynein motor function in neuronal migration and development. Genes \& development 15, 639-651, (2001). 
1053

1054

1055

1056

1057

1058

1059

1060

1061

1062

1063

1064

1065

1066

1067

1068

1069

1070

1071

1072

1073

1074

1075

1076

1077

1078

1079

1080

1081

1082

1083

1084

1085

1086

1087

1088

1089

1090

1091

1092

1093

1094

1095

1096

1097

16. Raaijmakers, J. A., Tanenbaum, M. E. \& Medema, R. H. Systematic dissection of dynein regulators in mitosis. J Cell Biol 201, 201-215, (2013).

17. Coquelle, F. M. et al. LIS1, CLIP-170's key to the dynein/dynactin pathway. Mol Cell Biol 22, 3089-3102, (2002).

18. Tsai, J. W., Bremner, K. H. \& Vallee, R. B. Dual subcellular roles for LIS1 and dynein in radial neuronal migration in live brain tissue. Nat Neurosci 10, 970-979, (2007).

19. Tsai, J. W., Chen, Y., Kriegstein, A. R. \& Vallee, R. B. LIS1 RNA interference blocks neural stem cell division, morphogenesis, and motility at multiple stages. $J$ Cell Biol 170, 935-945, (2005).

20. Yi, J. Y. et al. High-resolution imaging reveals indirect coordination of opposite motors and a role for LIS1 in high-load axonal transport. J Cell Biol 195, 193-201, (2011).

21. Chapman, D. E. et al. Regulation of in vivo dynein force production by CDK5 and 14-3-3epsilon and KIAA0528. Nature communications 10, 228, (2019).

22. Reddy, B. J. et al. Load-induced enhancement of Dynein force production by LIS1-NudE in vivo and in vitro. Nature communications 7, 12259, (2016).

23. McKenney, R. J., Vershinin, M., Kunwar, A., Vallee, R. B. \& Gross, S. P. LIS1 and NudE induce a persistent dynein force-producing state. Cell 141, 304-314, (2010).

24. Yamada, M. et al. LIS1 and NDEL1 coordinate the plus-end-directed transport of cytoplasmic dynein. EMBO J 27, 2471-2483, (2008).

25. Baumbach, J. et al. Lissencephaly-1 is a context-dependent regulator of the human dynein complex. eLife 6, (2017).

26. Gutierrez, P. A., Ackermann, B. E., Vershinin, M. \& McKenney, R. J. Differential effects of the dynein-regulatory factor Lissencephaly-1 on processive dyneindynactin motility. J Biol Chem 292, 12245-12255, (2017).

27. Jha, R., Roostalu, J., Cade, N. I., Trokter, M. \& Surrey, T. Combinatorial regulation of the balance between dynein microtubule end accumulation and initiation of directed motility. EMBO J 36, 3387-3404, (2017).

28. Huang, J., Roberts, A. J., Leschziner, A. E. \& Reck-Peterson, S. L. Lis1 acts as a "clutch" between the ATPase and microtubule-binding domains of the dynein motor. Cell 150, 975-986, (2012).

29. Toropova, K. et al. Lis1 regulates dynein by sterically blocking its mechanochemical cycle. eLife 3, (2014).

30. DeSantis, M. E. et al. Lis1 Has Two Opposing Modes of Regulating Cytoplasmic Dynein. Cell 170, 1197-1208 e1112, (2017).

31. Markus, S. M. \& Lee, W. L. Regulated offloading of cytoplasmic dynein from microtubule plus ends to the cortex. Dev Cell 20, 639-651, (2011).

32. Dix, C. I. et al. Lissencephaly-1 promotes the recruitment of dynein and dynactin to transported mRNAs. J Cell Biol 202, 479-494, (2013).

33. Wang, S. et al. Nudel/NudE and Lis1 promote dynein and dynactin interaction in the context of spindle morphogenesis. Mol Biol Cell 24, 3522-3533, (2013).

34. Marzo, M. G. et al. Molecular basis for dyneinopathies reveals insight into dynein regulation and dysfunction. bioRxiv, 635383, (2019). 
1098

1099

1100

1101

1102

1103

1104

1105

1106

1107

1108

1109

1110

1111

1112

1113

1114

1115

1116

1117

1118

1119

1120

1121

1122

1123

1124

1125

1126

1127

1128

1129

1130

1131

1132

1133

1134

1135

1136

1137

1138

1139

1140

1141

1142

35. Burgess, S. A., Walker, M. L., Sakakibara, H., Knight, P. J. \& Oiwa, K. Dynein structure and power stroke. Nature 421, 715-718, (2003).

36. Markus, S. M. \& Lee, W. L. Microtubule-dependent path to the cell cortex for cytoplasmic dynein in mitotic spindle orientation. Bioarchitecture 1, 209-215, (2011).

37. Curran, K. A. et al. Short Synthetic Terminators for Improved Heterologous Gene Expression in Yeast. ACS Synth Biol 4, 824-832, (2015).

38. Schmidt, H., Gleave, E. S. \& Carter, A. P. Insights into dynein motor domain function from a 3.3-A crystal structure. Nat Struct Mol Biol 19, 492-497, S491, (2012).

39. Carter, A. P., Cho, C., Jin, L. \& Vale, R. D. Crystal structure of the dynein motor domain. Science 331, 1159-1165, (2011).

40. Schmidt, H., Zalyte, R., Urnavicius, L. \& Carter, A. P. Structure of human cytoplasmic dynein-2 primed for its power stroke. Nature 518, 435-438, (2015).

41. Gibbons, I. R. et al. The affinity of the dynein microtubule-binding domain is modulated by the conformation of its coiled-coil stalk. J Biol Chem 280, 2396023965, (2005).

42. Kon, T. et al. Helix sliding in the stalk coiled coil of dynein couples ATPase and microtubule binding. Nat Struct Mol Biol 16, 325-333, (2009).

43. Urnavicius, L. et al. Cryo-EM shows how dynactin recruits two dyneins for faster movement. Nature 554, 202-206, (2018).

44. Lee, W. L., Oberle, J. R. \& Cooper, J. A. The role of the lissencephaly protein Pac1 during nuclear migration in budding yeast. J Cell Biol 160, 355-364, (2003).

45. Woodruff, J. B., Drubin, D. G. \& Barnes, G. Dynein-driven mitotic spindle positioning restricted to anaphase by She1p inhibition of dynactin recruitment. Mol Biol Cell 20, 3003-3011, (2009).

46. Markus, S. M. et al. Quantitative analysis of Pac1/LIS1-mediated dynein targeting: Implications for regulation of dynein activity in budding yeast. Cytoskeleton (Hoboken) 68, 157-174, (2011).

47. Lammers, L. G. \& Markus, S. M. The dynein cortical anchor Num1 activates dynein motility by relieving Pac1/LIS1-mediated inhibition. J Cell Biol 211, 309322, (2015).

48. Markus, S. M., Punch, J. J. \& Lee, W. L. Motor- and tail-dependent targeting of dynein to microtubule plus ends and the cell cortex. Curr Biol 19, 196-205, (2009).

49. Lee, W. L., Kaiser, M. A. \& Cooper, J. A. The offloading model for dynein function: differential function of motor subunits. J Cell Biol 168, 201-207, (2005).

50. Yin, H., Pruyne, D., Huffaker, T. C. \& Bretscher, A. Myosin V orientates the mitotic spindle in yeast. Nature 406, 1013-1015, (2000).

51. Hwang, E., Kusch, J., Barral, Y. \& Huffaker, T. C. Spindle orientation in Saccharomyces cerevisiae depends on the transport of microtubule ends along polarized actin cables. J Cell Biol 161, 483-488, (2003).

52. Liakopoulos, D., Kusch, J., Grava, S., Vogel, J. \& Barral, Y. Asymmetric loading of Kar9 onto spindle poles and microtubules ensures proper spindle alignment. Cell 112, 561-574, (2003). 
1143 53. Miller, R. K. \& Rose, M. D. Kar9p is a novel cortical protein required for cytoplasmic microtubule orientation in yeast. J Cell Biol 140, 377-390, (1998).

54. Ecklund, K. H. et al. She1 affects dynein through direct interactions with the microtubule and the dynein microtubule-binding domain. Nature communications 8, 2151, (2017).

55. Markus, S. M., Kalutkiewicz, K. A. \& Lee, W. L. She1-mediated inhibition of dynein motility along astral microtubules promotes polarized spindle movements. Curr Biol 22, 2221-2230, (2012).

1152 Redwine, W. B. et al. Structural basis for microtubule binding and release by

56. Redwine, W. B. et al. Structural basis for
dynein. Science 337, 1532-1536, (2012).

57. Sheeman, B. et al. Determinants of S. cerevisiae dynein localization and activation: implications for the mechanism of spindle positioning. Curr Biol 13,

1155

1156 364-372, (2003).

1157

1158

1159

58. Blake-Hodek, K. A., Cassimeris, L. \& Huffaker, T. C. Regulation of microtubule dynamics by Bim1 and Bik1, the budding yeast members of the EB1 and CLIP. 170 families of plus-end tracking proteins. Mol Biol Cell 21, 2013-2023, (2010).

1160

1161

1162

1163

1164

1165

1166

1167

1168

1169

1170

1171

1172

1173

1174

1175

1176

1177

1178

1179

1180

1181

1182

1183

1184

1185

1186

1187

1188

59. Faulkner, N. E. et al. A role for the lissencephaly gene LIS1 in mitosis and cytoplasmic dynein function. Nat Cell Biol 2, 784-791, (2000).

60. Duellberg, C. et al. Reconstitution of a hierarchical +TIP interaction network controlling microtubule end tracking of dynein. Nat Cell Biol, (2014).

61. Li, J., Lee, W. L. \& Cooper, J. A. NudEL targets dynein to microtubule ends through LIS1. Nat Cell Biol 7, 686-690, (2005).

62. Scoto, M. et al. Novel mutations expand the clinical spectrum of DYNC1H1associated spinal muscular atrophy. Neurology, (2015).

63. Poirier, K. et al. Mutations in TUBG1, DYNC1H1, KIF5C and KIF2A cause malformations of cortical development and microcephaly. Nature genetics 45, 639-647, (2013).

64. Willemsen, M. H. et al. Mutations in DYNC1H1 cause severe intellectual disability with neuronal migration defects. Journal of medical genetics 49, 179-183, (2012).

65. Bi, E. \& Pringle, J. R. ZDS1 and ZDS2, genes whose products may regulate Cdc42p in Saccharomyces cerevisiae. Mol Cell Biol 16, 5264-5275, (1996).

66. Knop, M. et al. Epitope tagging of yeast genes using a PCR-based strategy: more tags and improved practical routines. Yeast 15, 963-972, (1999).

67. Longtine, M. S. et al. Additional modules for versatile and economical PCRbased gene deletion and modification in Saccharomyces cerevisiae. Yeast 14, 953-961, (1998).

68. Markus, S. M., Omer, S., Baranowski, K. \& Lee, W. L. Improved Plasmids for Fluorescent Protein Tagging of Microtubules in Saccharomyces cerevisiae. Traffic 16, 773-786, (2015).

69. Song, S. \& Lee, K. S. A novel function of Saccharomyces cerevisiae CDC5 in cytokinesis. J Cell Biol 152, 451-469, (2001).

70. Weissmann, F. et al. biGBac enables rapid gene assembly for the expression of large multisubunit protein complexes. Proc Natl Acad Sci U S A 113, E25642569, (2016).

71. Gibson, D. G. et al. Enzymatic assembly of DNA molecules up to several hundred kilobases. Nat Methods 6, 343-345, (2009). 
1189 72. Kelley, L. A., Mezulis, S., Yates, C. M., Wass, M. N. \& Sternberg, M. J. The Phyre2 web portal for protein modeling, prediction and analysis. Nat Protoc 10, 845-858, (2015). is essential for dynein-dependent interactions of microtubules with the cortex. $J$ Cell Biol 151, 1337-1344, (2000). 